Document downloaded from:

http://hdl.handle.net/10251/38498

This paper must be cited as:

Braysy, O.; Martínez Molada, E.; Nagata, Y.; Soler Fernández, D. (2011). The mixed capacitated general routing problem with turn penalties. Expert Systems with Applications. 38(10):12954-12966. doi:10.1016/j.eswa.2011.04.092.

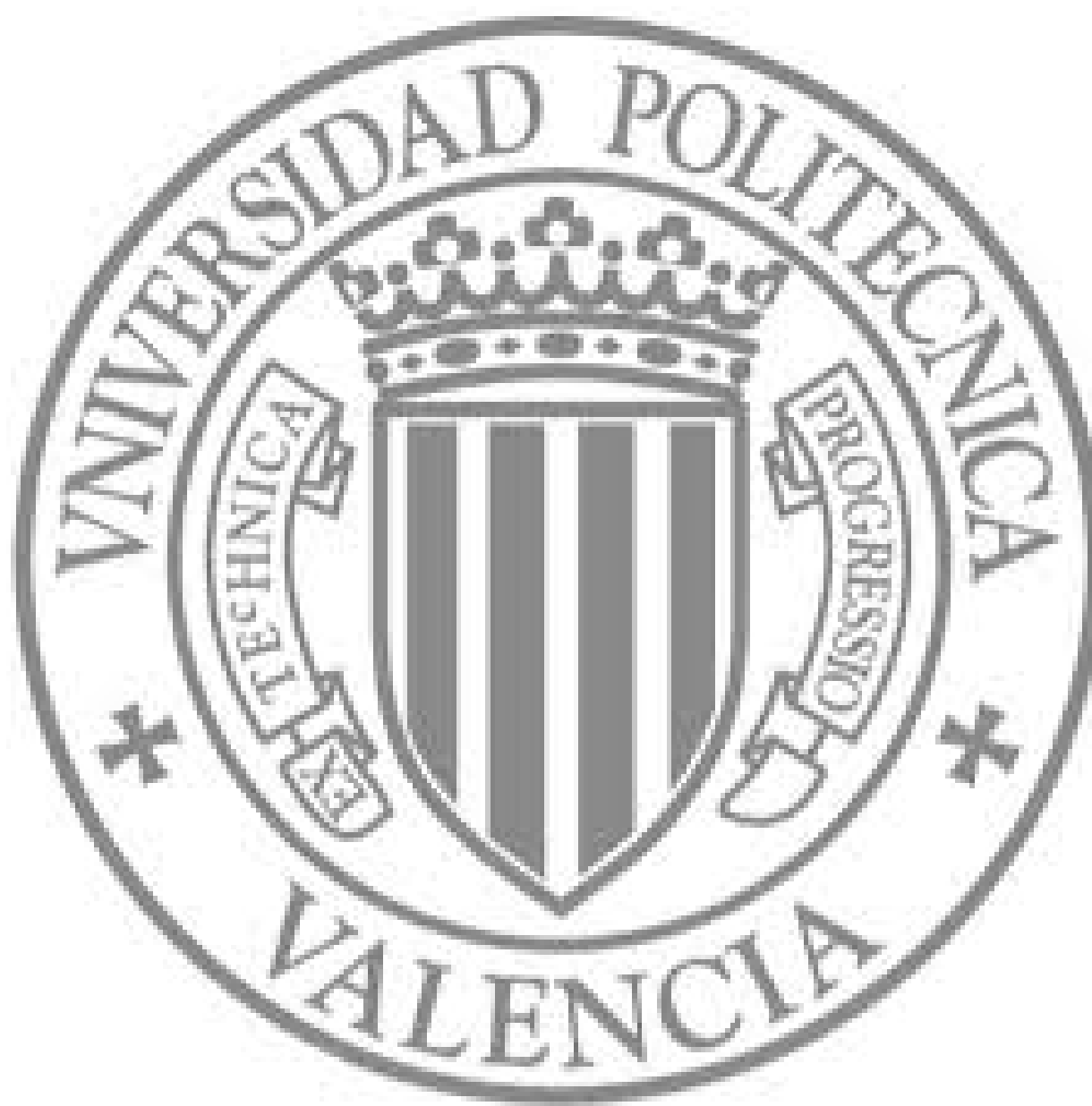

The final publication is available at

http://dx.doi.org/10.1016/j.eswa.2011.04.092

Copyright Elsevier 


\title{
The mixed capacitated general routing problem with turn penalties
}

\author{
Olli Bräysy ${ }^{1}$, Eulalia Martínez ${ }^{2}$, Yuichi Nagata ${ }^{3}$, David Soler ${ }^{2 *}$ \\ (1) Agora Innoroad Laboratory, Agora Center, P.O.Box 35, \\ FI-40014 University of Jyväskylä, Finland
}

(2) Instituto Universitario de Matemática Pura y Aplicada. Universidad Politécnica de Valencia Camino de Vera s/n, 46022, Valencia, Spain

(3) Interdisciplinary Graduate School of Science and Engineering, Tokyo Institute of Technology, 4259 Nagatsuta Midori-ku Yokohama, Kanagawa 226-8502, Japan

\begin{abstract}
In this paper we deal with the Mixed Capacitated General Routing Problem with Turn Penalties. This problem generalizes many important arc and node routing problems, and it takes into account turn penalties and forbidden turns, which are crucial in many real-life applications, such as mail delivery, waste collection and street maintenance operations. Through a polynomial transformation of the considered problem into a Generalized Vehicle Routing Problem, we suggest a new approach for solving this new problem by transforming it into an Asymmetric Capacitated Vehicle Routing Problem. In this way, we can solve the new problem both optimally and heuristically using existing algorithms. A powerful memetic algorithm and a set of 336 new benchmark instances are also

*Corresponding author: Address: Instituto Universitario de Matemática Pura y Aplicada. Universidad Politécnica de Valencia. Camino de Vera s/n, 46022, Valencia, Spain. Tel:+34 963877007-76667; fax:+34 963877669. E-mail addresses: olli.m.p.braysy@jyu.fi (O. Bräysy), eumarti@mat.upv.es (E. Martínez), nagata@fe.dis.titech.ac.jp (Y. Nagata), dsoler@mat.upv.es (D. Soler).
\end{abstract}


suggested. The experimental results show that the average deviation of the suggested solution method is less than 0.05 per cent with respect to optimum.

Keywords: Vehicle routing, capacitated general routing problem, turn penalties, transformation.

\section{Introduction}

In many real-life vehicle routing problems it is important to consider the risk and time associated with turns, i.e., the cost of the turn. Moreover, some turns, especially Uturns, can be forbidden. This last implies that a vehicle route made with a classical graph route generator may be illegal if it does not respect the traffic signals. For example, Figure 1 shows a traffic signal located in an arterial avenue from Valencia (Spain). It indicates that the next two left turns are forbidden and the way to dodge them. These forbidden turns cannot be taken into account if we model the city map with a classical graph.

Considering turn penalties and forbidden turns is particularly important in downtown areas and for large-size vehicles. But also turn penalties are important in order to save time in tours on foot. From Figure 2 it is easy to see that going on foot, right turn $a \rightarrow b$ can be considered with zero cost, while turn $a \rightarrow c$ is much more time-consuming, because it implies to cross two streets, with up to two traffic lights.

Usually the real-life vehicle routing software are based on separate modules for shortest path calculation and vehicle route optimization. The latter is based on given time and distance matrices, calculated typically in the beginning with the shortest path procedure. In this context, the distance and time calculation are based on fixed and known stopping points (usually addresses) for the vehicles and the possible turn penalties and forbidden turns are taken into account during the shortest path calculation. However, in practice, there are several applications where the exact stopping points are not known a priori and are part of the optimization problem, such as mail collection and delivery, waste collection and street maintenance operations. In these cases, including turn penalties and forbidden turns in the vehicle routing model is very important. 


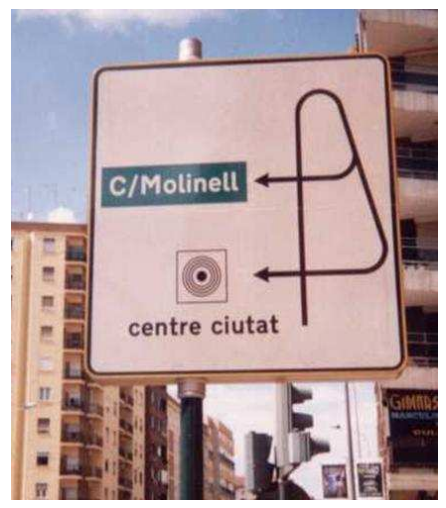

Figure 1. Traffic signal indicating two forbidden left turns.

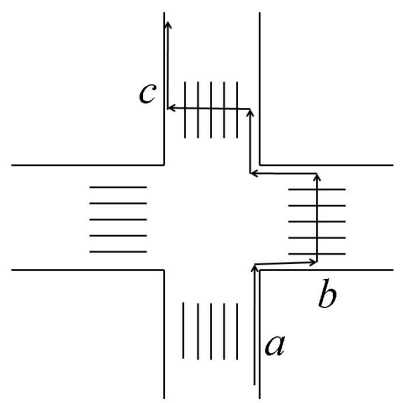

Figure 2. A street crossing.

So far research on extended vehicle routing models with turn penalties and forbidden turns has been scarce. For previous research, see Benavent and Soler (1999), Clossey et al (2001), Corberán et al (2002) and Soler et al (2008), that generalize several wellknown single vehicle routing problems to the existence of turn penalties. They provide theoretical results about complexity and resolution and/or computational results on these extensions. The last cited work studies the most general problem, the Mixed General Routing Problem (MGRP) with turn penalties, that includes all the cases studied in the previous cited papers. MGRP consists of finding a minimal cost closed walk on the links of a mixed graph $G$ which traverses a given subset of "required" links and a given subset of "required" vertices.

With respect to multivehicle routing problems, early papers that considered turn penalties focused on real-life applications and heuristic solution methods. See for example Bodin et al (1989) and Roy and Rousseau (1989). Later, turn penalties have been considered in the context of the Mixed Capacitated Arc Routing Problem (MCARP), see for example Bautista and Pereira (2004) and Belenguer et al (2006). The MCARP is an arc routing problem, in which a fleet of vehicles (with a known capacity) is based 
on a specified vertex (the depot) and must service a subset of the links of a mixed graph, with minimum total cost and such that the load assigned to each vehicle does not exceed its capacity and each link is serviced by exactly one vehicle.

Bautista et al (2008) present two ant colony metaheuristics for a real urban waste collection problem. This real problem is modeled as a particular case of the MCARP with turn penalties, in which they only consider two kind of turns: forbidden or allowed with zero cost. Finally, Perrier et al (2008) heuristically solve a real vehicle routing problem in the context of snow plowing operations that also takes into account the existence of forbidden turns.

The multivehicle extension (with capacity constraints) of the MGRP is called the Mixed Capacitated General Routing Problem (MCGRP). Due to its complexity, there are only few works on MCGRP, see e.g. Jansen (1993) who studied the undirected case and Pandit and Muralidharan (1995). However, particular cases of the MCGRP, such as the capacitated arc routing problem (CARP) and the capacitated vehicle routing problem (CVRP) have attracted a huge amount of research.

In this paper we present a generalization of the MCGRP that considers turn penalties and forbidden turns. The objective is to minimize the sum of the costs of the traversed arcs and edges together with the penalties associated with the turns made. We call the new problem the Mixed Capacitated General Routing Problem with Turn Penalties (MCGRPTP).

As far as we know, this is the first time that MCGRPTP is presented in the literature. Moreover, there is no previous research on multivehicle node routing problems with turn penalties. In this paper we also present for the first time an approach for solving capacitated routing problems with turn penalties, through suggesting a new polynomial transformation from the MCGRPTP to an asymmetric CVRP (ACVRP). To be more precise, the transformation is done in two steps: we first transform the MCGRPTP into a generalized VRP (GVRP), using a new approach suggested in this paper. The key idea of GVRP, compared to CVRP is that in GVRP each customer has several alternative service locations, and only one of them has to be selected for service. For more details on GVRP, see e.g. Ghiani and Improta (2000). In the second step we transform the GVRP into an ACVRP, using a model presented in Soler et al (2009). 
Finally, we present a set of new benchmark problems and a very powerful memetic algorithm for the ACVRP, based on a previous study by Nagata and Bräysy (2009). The experimental results show an average deviation equal to $0.05 \%$ for instances with known optimal solution and that large-size problems can be solved with the suggested MA.

The suggested transformation makes it possible to use also any other powerful algorithm developed for ACVRP, see e.g. Fischetti et al (1994), Vigo (1996) or the more recent heuristic by De Franceschi et al (2006).

The remainder of this paper is organized as follows. Section 2 introduces some definitions and notations in order to formally define and solve the MCGRPTP. In Section 3, through two transformations, we prove that the MCGRPTP can be transformed in polynomial time into a Generalized Vehicle Routing Problem (GVRP). It is known (Soler et al (2009)) that the GVRP can be transformed into an ACVRP, so in Section 4 we show computational results for several sets of ACVRP benchmarks. Finally, in Section 5 we present our conclusions.

\section{Definitions and notations}

First, to our aim, we formally define two known problems cited before: the Asymmetric Capacitated Vehicle Routing Problem (ACVRP) and the Generalized Vehicle Routing Problem (GVRP). The second one is an extension of the ACVRP, introduced by Ghiani and Improta (2000), that can model several real-world situations and that will be the "cornerstone" to solve our problem:

The ACVRP is defined as follows:

Let $G=(V, A)$ be a complete digraph, $V=\left\{v_{i}\right\}_{i=0}^{n}$ being its set of vertices, where $v_{0}$ is the depot vertex. Each vertex $v_{i}$ with $i>0$ has an associated demand $d_{i}>0$ and each $\operatorname{arc}\left(v_{i}, v_{j}\right) \in A$ has an associated cost $c_{i, j} \geq 0$. Moreover, a fleet of $k$ vehicles with the same capacity $W$ is available at the depot.

Find a set of $k$ shortest routes, each starting and ending at the depot, such that each vertex $v_{i}(\forall i \in\{1, \ldots, n\})$ must be visited by one and only one vehicle and the sum of the demands of the vertices visited by each vehicle does not exceed its capacity $W$. 
As in other papers cited in the introduction, in all the capacitated routing problems discussed here, we will consider $k$ to be equal to the minimum number of vehicles needed to serve all demands.

The GVRP is defined as follows:

Let $G=(V, A)$ be a directed graph where the vertex set $V$ is partitioned into $m+1$ nonempty subsets $S_{0}, S_{1}, \ldots, S_{m}$ such that $S_{0}$ has only one vertex $v_{0}$ (the depot), $S_{h}$ $(h=1, \ldots, m)$ represents $l(h)$ possible locations of the same vertex which has associated a positive demand $d_{h}$, and each arc $\left(v_{i}, v_{j}\right) \in A$ has associated a cost $c_{i, j} \geq 0$. Moreover, a fleet of $k$ homogeneous vehicles having the same capacity $W$ is available at the depot.

Find a set of $k$ shortest routes, each starting and ending at the depot, such that each subset $S_{h}(h=1, \ldots, m)$ is visited exactly once and the sum of the demands of every route does not exceed the capacity $W$ of the vehicle.

Next, we need to show some concepts and notations that have been used in previous works on turn penalties:

Given a mixed graph $G=(V, E, A)$, each pair of links $a=(u, v), b=(v, w) \in E \cup A$ has an associated turn at $v$, based on going from $a$ to $b$, denoted as $[a b]$. Moreover, if $a, b \in E$, the same pair has another associated turn at $v$, based on going from $b$ to $a$, denoted as $[b a]$. Each edge $e$ incident with $v$ has an associated U-turn at $v$ that, if necessary, will be denoted by [eve]. Each link in $G$ has associated a nonnegative cost and each allowed turn in $G$ has associated a nonnegative penalty.

Given $a=(u, v), b=(s, t) \in E \cup A$, a $v$-s feasible chain from $a$ to $b$ is an alternating sequence of links and allowed turns $\left\{a_{1},\left[a_{1} a_{2}\right], a_{2}, \ldots,\left[a_{r-1} a_{r}\right], a_{r},\left[a_{r} b\right]\right\}$, where $a_{1}=a$. The cost of a feasible chain is defined as the sum of the costs of the arcs it traverses plus the sum of the penalties of the turns it makes. A $v$-s feasible chain from $a$ to $b$ is closed if $a=b$ and $s \neq v$.

Given $a=(u, v), b=(s, t) \in E \cup A$, a shortest (minimum cost) $v$-s feasible chain from $a$ to $b$ will be denoted by s.f.c. $\left(v^{a}, s^{b}\right)$.

Note that a feasible chain is defined such that it begins at a link and ends at a turn. This is very important in the context of forbidden or penalized turns. In classical routing problems, if we have to go from a vertex $u$ to a vertex $v$ and then to a vertex $w$, we only have to connect the shortest path from $u$ to $v$ with the shortest path from $v$ 
to $w$. But even if these shortest paths have been constructed taking into account turn conditions, the connection of both paths at $v$ can give rise to an unavoidable forbidden turn (U-turn for example). In our case, the connection between two feasible chains at a vertex $v$ is possible only if the first one ends at a turn $[(t, v)(v, s)]$ and the second one begins at the link $(v, s)$, which avoids the existence of forbidden turns. Therefore, we cannot use paths between vertices as in the classical way, and this increases the difficulty of modeling how to serve demands at vertices, specially if these vertices have undesirable turns.

With these previous concepts we can formally define the problem that we study in this paper. The Mixed Capacitated General Routing Problem with Turn Penalties (MCGRPTP) is defined as follows:

Let $G=(V, E, A)$ be a mixed graph where each link $(i, j) \in E \cup A$ has an associated cost $c_{i j} \geq 0$ and each turn $[a b]$ has an associated penalty $p_{[a b]} \geq 0\left(p_{[a b]}=+\infty\right.$ if turn $[a b]$ is forbidden). One of the vertices, say $v_{0}$, represents the depot where there are $k$ vehicles of an identical capacity $W>0$ and in it all the turns are allowed with zero penalty. Let $R \subseteq E \cup A$ be a set of required links such that each $(i, j) \in R$ has an associated positive demand $q_{i j} \leq W$, and let $V_{R} \subseteq V-\left\{v_{0}\right\}$ be a set of required vertices such that each $v \in V_{R}$ has an associated positive demand $q_{v} \leq W$.

Find $k$ closed feasible chains in $G$, one for each vehicle, that minimize the total cost and such that each chain passes through the depot, each demand is served by only one vehicle and the total demand served by each vehicle does not exceed its capacity $W$.

Note that allowing all the turns with zero penalties at the depot is due to the fact that in real-world situations, the depot normally represents a warehouse from which the vehicles begin their journey and to which they return. It makes no sense considering forbidden/penalty turns in the warehouse as the truck leaves from depot independently of the route the truck made before. Moreover, these warehouses are usually placed outside the cities with good road communications and of easy access.

Hereinafter and as in similar papers, each non-required edge will be replaced by two arcs of the same cost and opposite direction; then we assume that all edges in the graph are required $\left(E_{R}=E\right.$, with $\left.R=E_{R} \cup A_{R}\right)$. Finally, for simplicity, we will not write 
the middle turns of a feasible chain. For example, the feasible chain $\{a,[a b], b,[b, c]\}$ will be written as $\{a, b,[b, c]\}$.

In the particular case of the MCGRPTP in which $V_{R}=\emptyset$, we have the MCARP with turn penalties, and in the particular case of the MCGRPTP in which $k=1$ we have the MGRP with turn penalties. Therefore, the problem presented here generalizes both the single vehicle and the multivehicle routing problems with turn penalties studied in the literature.

\section{Solving the MCGRPTP}

To solve the MCGRPTP, we will first transform it into a GVRP, which in turn can be transformed into an ACVRP.

\subsection{Transformation of the MCGRPTP into a GVRP}

Let $G=(V, E, A)$ be a mixed graph where a MCGRPTP is defined, $E \cup A_{R}$ being the required link set and $V_{R}$ the required vertex set. Due to the fact that in the GVRP the demand is at the vertices, we will transform graph $G$ in which we have defined the MCGRPTP into a directed graph $G^{*}=\left(V^{*}, A^{*}\right)$ such that the vertices in $G^{*}$ are related with the required links and required vertices in $G$.

To this aim, we first construct an intermediate directed graph $G^{\prime}=\left(V^{\prime}, A^{\prime}\right)$ from $G$ as follows:

First, each required edge is replaced in $G$ by two opposite required arcs, both with the edge cost and the edge demand.

Second, subset $V_{R}$ is partitioned into two subsets, $V_{R_{1}}$ and $V_{R_{2}}$, such that vertices containing all allowed zero-penalty turns belong to $V_{R_{1}}$, and vertices containing forbidden or positive-penalty turns belong to $V_{R_{2}}$.

For each $v \in V_{R_{1}} \cup\left\{v_{0}\right\}$, replace vertex $v$ in $G$ by two vertices $v^{e}$ and $v^{l}$, so that $v^{e}$ has only entering arcs (the arcs entering at $v$ ) and $v^{l}$ has only leaving arcs (the arcs leaving from $v$ ). Add a required arc $a_{v}=\left(v^{e}, v^{l}\right)$ to $G$ such that all turns at $v^{e}$ and $v^{l}$ are allowed with zero penalty and the demand of this arc in $G^{\prime}$ is the one of the required vertex $v$ in $G$ (the demand corresponding to the arc from the depot vertex is 
obviously zero). Note that traversing $\operatorname{arc} a_{v}$ in $G^{\prime}$ is then equivalent to passing through vertex $v$ in the original graph $G$.

For each $v \in V_{R_{2}}$ do:

- Replace vertex $v$ in $G$ by the same number of vertices $v_{i j}$ as those of allowed turns $\left[a_{i} b_{j}\right]$ at $v$, so that each of these copies has only one entering arc $\left(a_{i}\right)$ and one leaving arc $\left(b_{j}\right)$, with its corresponding allowed turn. Note that if $a_{i}$ is an entering arc at $v, G^{\prime}$ will contain at least as many copies of the entering arc $a_{i}$ as there are allowed turns involving $a_{i}$ at $v$. The same applies to the leaving $\operatorname{arc} b_{j}$ from $v$.

- Then, replace each vertex $v_{i j}$ by two vertices, $v_{i j}^{e}$ and $v_{i j}^{l}$, and add a "required" $\operatorname{arc} a_{v_{i j}}=\left(v_{i j}^{e}, v_{i j}^{l}\right)$ between them with cost zero, such that $p_{\left[a_{i} a_{v_{i j}}\right]}=p_{\left[a_{i} b_{j}\right]}$ and $p_{\left[a_{v_{i j}} b_{j}\right]}=0$, i.e. the penalty that was in the turn at $v_{i j}$ is moved to vertex $v_{i j}^{e}$, and all these arcs will have the same demand, the one of the required vertex $v$. Note that traversing only one of these required arcs $a_{v_{i j}}$ in $G^{\prime}$ involves passing through vertex $v$ in $G$.

Note that in the last paragraph we have written required in quotes because for each $v \in V_{R_{2}}$, only one of the generated arcs must be served.

After this transformation we have a directed graph $G^{\prime}=\left(V^{\prime}, A^{\prime}\right)$ such that the subset $A_{R}^{\prime}$ comes from the required arcs, required edges and required vertices in $G$. $A_{R}^{\prime}$ will give rise to a partitioned set of vertices $V^{*}$ in the graph $G^{*}$ in which we will define the GVRP. Each arc between two of these vertices that do not form part of the same subset, will have associated the cost of the shortest feasible chain between the two corresponding links in $G^{\prime}$.

From $G^{\prime}$ we then construct the graph $G^{*}=\left(V^{*}, A^{*}\right)$ as follows:

- For each arc $a_{v} \in A_{R}^{\prime}$ with $v \in V_{R_{1}} \cup\left\{v_{0}\right\}$, associate a vertex set $S_{v}=\left\{x_{a_{v}}\right\}$ in $G^{*}$, with its corresponding demand in $x_{a_{v}}$.

- For each $v \in V_{R_{2}}$, associate a vertex set $S_{v}$ in $G^{*}$ with as many vertices $x_{a_{v_{i j}}}$ as $\operatorname{arcs} a_{v_{i j}}$ are in $A_{R}^{\prime}$, all of them with the same corresponding demand. 
- For each arc $a \in A_{R}^{\prime}$ that comes from a required arc in $G$, associate a vertex set $S_{a}$ in $G^{*}$ with as many copies of a vertex $x_{a}$ as copies of arc $a$ appear in $G^{\prime}$, all of them with the same corresponding demand.

- For each pair of opposite required $\operatorname{arcs} e_{1}, e_{2} \in A_{R}^{\prime}$ that come from a required edge $e$ in $G$, associate a vertex set $S_{e}$ in $G^{*}$ with as many copies of vertices $x_{e_{1}}$ and $x_{e_{2}}$ as copies of $\operatorname{arcs} e_{1}$ and $e_{2}$ respectively appear in $G^{\prime}$, all of them with the same corresponding demand.

- For each pair of vertices $x_{a}, x_{b} \in V^{*}$ with $x_{a} \in S_{i}, x_{b} \in S_{j}, i \neq j$ being $a=(u, v)$ and $b=(s, t)$, add $\operatorname{arcs}\left(x_{a}, x_{b}\right)$ and $\left(x_{b}, x_{a}\right)$ to $A^{*}$, with the cost of the s.f.c. $\left(v^{a}, s^{b}\right)$ and of the s.f.c. $\left(t^{b}, u^{a}\right)$ respectively in $G^{\prime}$ (if they exist).

- There is no arc between vertices belonging to the same $S_{i}$.

Given an MCGRPTP in $G$, we define a GVRP in $G^{*}$ where the vertex set $V^{*}$ is partitioned into the following subsets: $S_{v}$ for all $v \in V_{R_{1}} \cup\left\{v_{0}\right\}$ (hereinafter we will denote the subset corresponding to the depot by $\left.S_{0}=\left\{v_{D}\right\}\right), S_{v}$ for all $v \in V_{R_{2}}, S_{a}$ for all $a \in A_{R}$ and $S_{e}$ for all $e \in E_{R}$.

Let us see an example of the construction of the auxiliary graph $G^{*}$. Consider the mixed graph given in Figure 3 corresponding to an MCGRPTP in which vertex 1 represents the depot, there are two vehicles both with capacity 55, and there are three required $\operatorname{arcs} a, b$ and $c$, with demands 25, 25 and 20 respectively, a required edge $e$ with demand 20 and a required vertex 5 with demand 10 . Then the total demand in the graph is 100 units. Link costs and demands appear in Figure 3 with different size (demands appear in bold and small size).

We will suppose in this graph that all U-turns are forbidden except at vertex 1 (the depot) at which all turns are allowed with penalty zero, and in the rest of the vertices, right turns (according to the drawing of the graph) have penalty 1, left turns have penalty 3 except for the turn from arc $b$ to arc $(2,1)$ that is considered forbidden, and going straight ahead, as it occurs in the turn from arc $a$ to edge $e$, has penalty zero. 


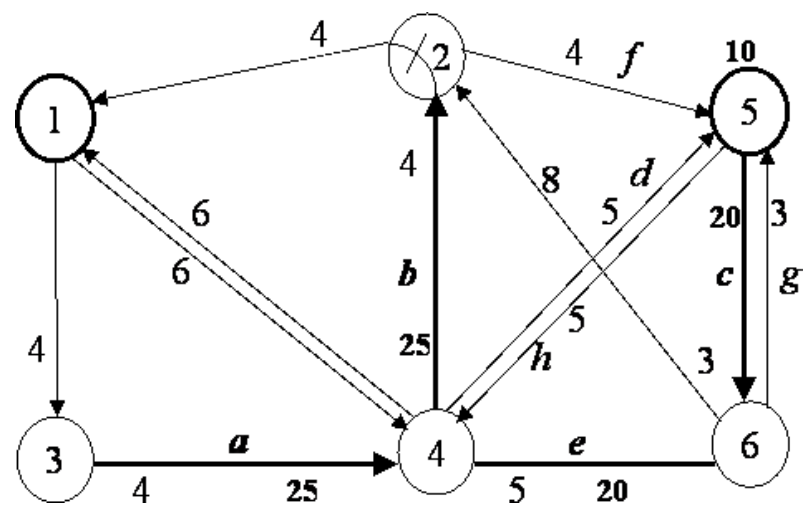

Figure 3. Graph $G$ with $A_{R}=\{a, b, c\}, E_{R}=\{e\}$ and $V_{R}=\{5\}$.

Starting from the information given by the initial graph $G=(V, E, A)$, where $A_{R}=\left\{(3,4),(4,2),((5,6)\}, E_{R}=\{(4,6)\}, V_{R}=\{5\}\right.$ and the depot is vertex 1 , we construct the intermediate graph $G^{\prime}$ (see Figure 4):

First, we replace the required edge $e=(4,6)$ with demand $q_{e}=20$ by two opposite $\operatorname{arcs} e_{1}, e_{2}$ with the same cost 5 and the same demand 20 .

Second, we replace vertex 1 by the sequence $\left\{1^{e}, a_{1}, 1^{l}\right\}, a_{1}$ with cost zero.

Finally, we transform vertex 5 , which belongs to $V_{R_{2}}$ (here $V_{R_{1}}=\emptyset$ ), into four arcs, as many as allowed turns in it, all of them with cost zero and demand 10.

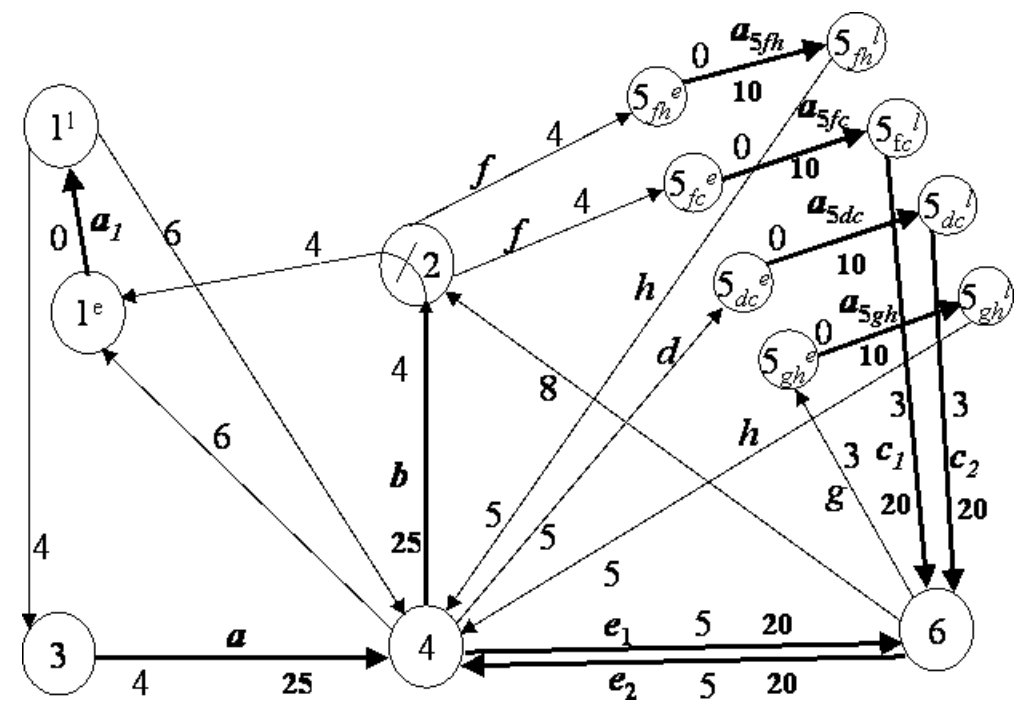

Figure 4. Intermediate graph $G^{\prime}$.

From the intermediate graph $G^{\prime}=\left(V^{\prime}, A^{\prime}\right)$ we can already construct the directed graph $G^{*}=\left(V^{*}, A^{*}\right)$.

The vertex set $V^{*}$ is given by the following partition: 
- A subset $S_{0}$ with only one vertex $x_{D}$ representing the depot and corresponding to the required $\operatorname{arc} a_{1}$.

- Subsets $S_{a}$ and $S_{b}$, each one with only one vertex $x_{a}$ and $x_{b}$ respectively, for the required $\operatorname{arcs} a=(3,4)$ and $b=(4,2)$, both with demand 25 , and subset $S_{c}$ with two vertices $x_{c_{1}}$ and $x_{c_{2}}$, both with demands 20 , for the required $\operatorname{arc} c=(5,6)$ which have two copies in $G^{\prime}$.

- Subset $S_{e}$ with two vertices $x_{e_{1}}$ and $x_{e_{2}}$ both with demand 20, for the required edge $e=(4,6)$.

- Subset $S_{5}$ with four vertices $x_{5_{f h}}, x_{5_{f c}}, x_{5_{d c}}$ and $x_{5_{g h}}$ all of them with demand 10, for the required vertex 5 in $V_{R_{2}}$.

Figure 5 shows graph $G^{*}$ in which, for simplicity, each pair of arcs $\left(x_{r}, x_{t}\right)$ and $\left(x_{t}, x_{r}\right)$ with $x_{r} \in S_{i}, x_{t} \in S_{j}$ and $i \neq j$ has been drawn as a line with two arrow heads, one at each end, and the arc costs (normally different for each direction) have been omitted.

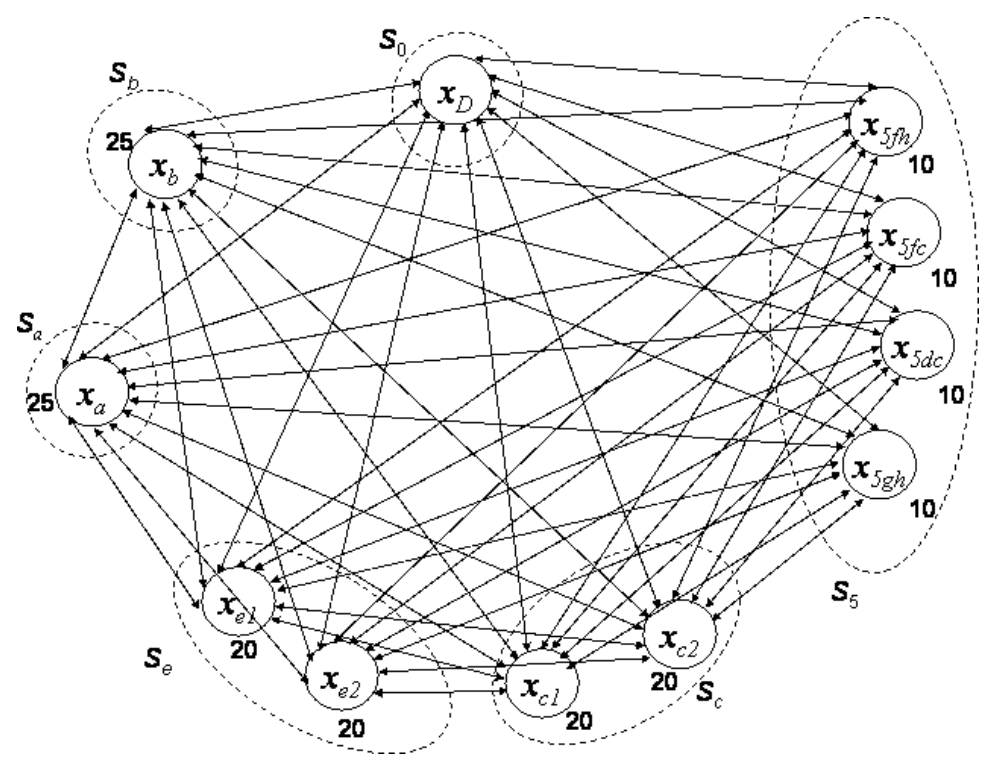

Figure 5. Directed graph $G^{*}$ associated with $G$.

Theorem 1 An MCGRPTP defined in G can be transformed in polynomial time into the corresponding GVRP defined in $G^{*}$. 
Proof. By the construction of $G^{\prime}$, given $B=\left\{T_{i}\right\}_{i=1}^{k}$ a set of $k$ feasible closed chains in $G$ corresponding to a solution to the MCGRPTP, we can associate with $B$ a set $B^{\prime}=\left\{T_{i}^{\prime}\right\}_{i=1}^{k}$ of $k$ feasible closed chains in $G^{\prime}$ such that for all $i \in\{1, \ldots, k\}$ we have:

- $T_{i}^{\prime}$ traverses arc $a_{1}$ (corresponding to the depot node 1 in $G$ ).

- $T_{i}$ passes through a vertex $v \in V_{R_{1}}$ iff $T_{i}^{\prime}$ traverses arc $a_{v}$ in $G^{\prime}$.

- $T_{i}$ passes through a vertex $v \in V_{R_{2}}$ iff $T_{i}^{\prime}$ traverses an arc $a_{v_{i j}}$.

- $T_{i}$ traverses arc $a \in A_{R}$ iff $T_{i}^{\prime}$ traverses a copy of arc $a$ in $G^{\prime}$.

- $T_{i}$ traverses edge $e \in E$ iff $T_{i}^{\prime}$ traverses a copy of arc $e_{1}$ or a copy of arc $e_{2}$ in $G^{\prime}$.

- $T_{i}^{\prime}$ has the same cost as $T_{i}$.

- Moreover, we will suppose that if $T_{i}$ satisfies demand at $v \in V_{1}\left(v \in V_{2}\right)\left(a \in A_{R}\right)$ $(e \in E)$, then $T_{i}^{\prime}$ satisfies the demand located at $a_{v}$ (one and only one arc $a_{v_{i j}}$ ) (one and only one copy of arc $a$ in $G^{\prime}$ ) (one and only one copy of $e_{1}$ or $e_{2}$ in $G^{\prime}$ ).

Summarizing, for all $i \in\{1, \ldots, k\} T_{i}^{\prime}$ is a feasible closed chain in $G^{\prime}$ that traverses $a_{1}$, satisfies the same demands as $T_{i}$ and has the same cost as $T_{i}$.

Once we have constructed $B^{\prime}$ from $B$, for each $i \in\{1, \ldots, k\}$, from $T_{i}^{\prime}$ we construct a cycle $C_{i}^{B}$ in $G^{*}$ as follows:

Suppose that $T_{i}^{\prime}$ satisfies, in this chronological order, the demands $q_{j_{1}}, q_{j_{2}}, \ldots, q_{j_{m_{i}}}$. Then $C_{i}^{B}$ is a cycle in $G^{*}$ that visits, in this order, the sets of vertices $S_{0}, S_{j_{1}}, S_{j_{2}}, \ldots$, $S_{j_{m_{i}}}, S_{0}$, and for all $t \in\left\{1, \ldots, m_{i}\right\}, C_{i}$ visits only the vertex at $S_{t}$ coming from the arc in $G^{\prime}$ at which demand has been satisfied by $T_{i}^{\prime}$.

It is evident that the set of cycles $L^{B}=\left\{C_{i}^{B}\right\}_{i=1}^{k}$ is a solution to the GVRP in $G^{*}$ its cost $c^{*}\left(L^{B}\right)$ being less than or equal to $c(B)$, this last due to the fact that the cost of the route segment of one feasible closed chain $T_{i}^{\prime}$ in $G^{\prime}$ between two consecutive serviced arcs (including the depot arc $a_{1}$ ) is greater than or equal to the cost of the shortest feasible chain in $G^{\prime}$ between them (from the first serviced one to the second serviced one), while the cost of the arc in $C_{i}^{B}$ in $G^{*}$ from the vertex coming from a 
serviced arc in $G^{\prime}$ to the vertex coming from the next serviced arc in $G^{\prime}$ is equal to the one of the shortest feasible chain in $G^{\prime}$ between them.

On the other hand, let $L=\left\{C_{i}\right\}_{i=1}^{k}$ be a set of $k$ cycles corresponding to a solution to the GVRP in $G^{*}$. For each $i \in\{1, \ldots, k\}$, from $C_{i}$ we construct a feasible closed chain $T_{i}^{L}$ in $G^{\prime}$ as follows:

Let $\left(x_{a}, x_{b}\right)$ be a generic arc of $C_{i}, a=(u, v)$ and $b=(w, r)$ being the $\operatorname{arcs}$ in $A_{R}^{\prime}$ from which vertices $x_{a}$ and $x_{b}$ come, respectively. Arc $\left(x_{a}, x_{b}\right)$ will give rise in $T_{i}^{L L}$ to the s.f.c. $\left(v^{a}, w^{b}\right)$, and such that $T_{i}^{L}$ assumes the demand at $a$ (the same as the one in $x_{a}$ ) and $b$ (the same as the one in $x_{b}$ ). Note that in this way, $T_{i}^{\prime L}$ has the same cost as $C_{i}$ and satisfies the same demands as $C_{i}$.

From the set $B^{\prime L}=\left\{T_{i}^{L L}\right\}_{i=1}^{k}$ of $k$ feasible closed chains in $G^{\prime}$, we construct now a set $B^{L}=\left\{T_{i}^{L}\right\}_{i=1}^{k}$ of $k$ feasible closed chains in $G$ as follows:

- "Contract" each sequence in $T_{i}^{L}$ of the form $\left(u, v^{e}\right)\left(v^{e}, v^{l}\right)\left(v^{l}, w\right)$ with $a_{v}=\left(v^{e}, v^{l}\right)$ if $v \in V_{R_{1}} \cup\{1\}$ by $(u, v)(v, w)$ in $T_{i}^{L}$.

- "Contract" each sequence in $T_{i}^{L}$ of the form $\left(u, v_{i j}^{e}\right)\left(v_{i j}^{e}, v_{i j}^{l}\right)\left(v_{i j}^{l}, w\right)$ with $a_{v_{i j}}=$ $\left(v_{i j}^{e}, v_{i j}^{l}\right)$ if $v \in V_{R_{2}}$ by $(u, v)(v, w)$ in $T_{i}^{L}$.

- If $T_{i}^{L}$ traverses a copy of $\operatorname{arc} e_{1}$ or a copy of $\operatorname{arc} e_{2}$ in $G^{\prime}$, with $e \in E$, replace this copy in $T_{i}^{L}$ by edge $e$.

- Any other link or turn in $T_{i}^{L}$ is replaced by itself in $T_{i}^{L}$.

- Demand at $v \in V_{1}\left(v \in V_{2}\right)\left(a \in A_{R}\right)(e \in E)$ is assigned to route $T_{i}^{L}$ iff $T_{i}^{L}$ satisfies the demand located at $a_{v}$ (one and only one arc $a_{v_{i j}}$ ) (one and only one copy of arc $a$ in $G^{\prime}$ ) (one and only one copy of $e_{1}$ or $e_{2}$ in $G^{\prime}$ ).

It is evident that $B^{L}=\left\{T_{i}^{L}\right\}_{i=1}^{k}$ is a solution to the MCGRPTP in $G$ with $c\left(B^{L}\right)=$ $c^{*}(L)$, this last due to the fact that for all $i, c\left(T_{i}^{L}\right)=c^{\prime}\left(T_{i}^{\prime L}\right)$.

Let then $L^{\text {opt }}$ be an optimal GVRP solution in $G^{*}$ and let $B^{L^{\text {opt }}}$ be the MCGRPTP solution in $G$ obtained from $L^{\text {opt }}$ as described above. For each MCGRPTP solution $B$ in $G$ we have:

$$
c(B) \geq c^{*}\left(L^{B}\right) \geq c^{*}\left(L^{o p t}\right)=c\left(B^{L^{o p t}}\right)
$$

Therefore, $B^{L^{\text {opt }}}$ is an optimal MCGRPTP in $G$. I 


\subsection{Solving the GVRP in $G^{*}$ through an ACVRP}

Once we have transformed our MCGRPTP into a GVRP defined in $G^{*}=\left(V^{*}, A^{*}\right)$, from $G^{*}$ we construct a digraph $\hat{G}=(\hat{V}, \hat{A})$ as follows:

$-\hat{V}=V^{*}$.

- For each $S_{i}$ with $i \in\{0,1, \ldots, m\}$ and $\left|S_{i}\right|>1$, order its vertices consecutively in an arbitrary way $\left\{v_{1}^{i}, \ldots, v_{l(i)}^{i}\right\}$; then, for $j=1, \ldots, l(i)-1$, define the cost of $\operatorname{arc}\left(v_{j}^{i}, v_{j+1}^{i}\right) \in \hat{A}$ as zero; also define the cost of arc $\left(v_{l(i)}^{i}, v_{1}^{i}\right)$ as zero.

- For every $v_{j}^{i} \in S_{i}$ and every $w \notin S_{i}$ define the cost of arc $\left(v_{j}^{i}, w\right)$ in $\hat{A}$ equal to the cost in $G^{*}$ of the arc $\left(v_{j+1}^{i}, w\right)\left(\left(v_{1}^{i}, w\right)\right.$ if $\left.j=l(i)\right)$ plus a fixed positive large quantity $M$ if $\left|S_{i}\right|>1$, and equal to the cost in $G^{*}$ of $\operatorname{arc}\left(v_{j}^{i}, w\right)$ plus $M$ if $\left|S_{i}\right|=1$.

- Any other arc in $\hat{A}$ has infinite cost.

- Assign positive demands having sum equal to $d_{i}$ to the vertices in $S_{i} \forall i$, except for the depot subset.

As it was proved by Soler et al (2009), the GVRP can be transformed into an ACVRP. Following their proof, we can see that to solve the GVRP in $G^{*}$ we can solve the ACVRP in the digraph $\hat{G}$, and to obtain a GVRP solution from an ACVRP one, we just have to identify each path in the ACVRP solution $\left(v_{j}^{i}, v_{j+1}^{i}, \ldots, v_{l(i)}^{i}, v_{1}^{i}, \ldots, v_{j-1}^{i}, w\right)$ $w \notin S_{i}$ ( $w$ can be the depot) if $j \neq 1$ and $\left|S_{i}\right|>1$, or $\left(v_{1}^{i}, \ldots, v_{l(i)}^{i}, w\right)$ if $j=1$ and $\left|S_{i}\right|>1$, or $\left(v_{j}^{i}, w\right)$ if $\left|S_{i}\right|=1$ (in this last case $v_{j}^{i}$ can be the depot), with the arc $\left(v_{j}^{i}, w\right)$ in $G^{*}$. An optimal ACVRP solution $H_{o p t}$ in $\hat{G}$, will give rise to an optimal GVRP solution $L_{\text {opt }}$ in $G^{*}$ with cost $c^{*}\left(L_{\text {opt }}\right)=\hat{c}\left(H_{\text {opt }}\right)-M(m+k)$.

Going on with our example, from the graph $G^{*}$ where the GVRP is defined (Figure 5) we define the ACVRP in the digraph $\hat{G}$ (see Figure 6) where, for simplicity again, the pairs of $\operatorname{arcs}\left(x_{r}, x_{t}\right)$ and $\left(x_{t}, x_{r}\right)$ with $x_{r} \in S_{i}, x_{t} \in S_{j}$ and $i \neq j$ have been drawn as lines with two arrow heads, one at each end, and the arc costs (normally different for each direction) have been omitted. Figure 6 shows the cost zero "intraset" arcs and the demand assigned to each vertex. For example, vertex 5 , belonging to $V_{R_{2}}$ and 
with demand 10 in $G$, has associated the set $S_{5}$ in $G^{*}$ with four vertices that in $\hat{G}$ have demands 2, 2, 3 and 3, respectively.

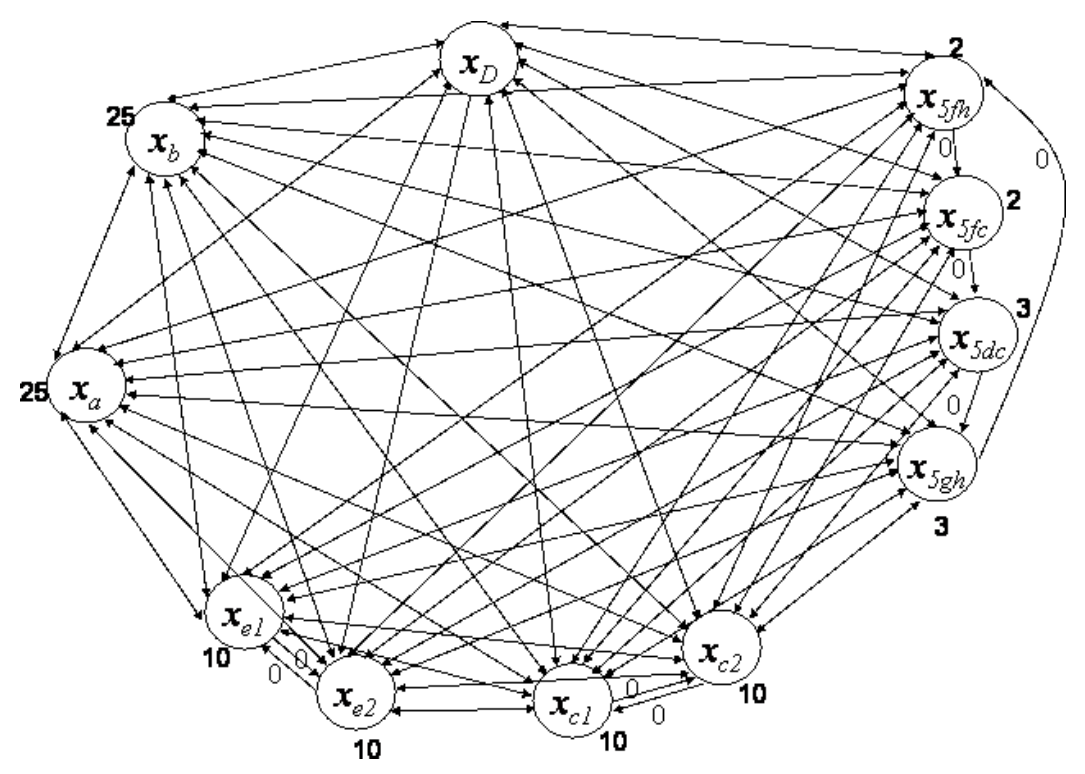

Figure 6. Directed graph $\hat{G}$ associated with $G^{*}$.

Figure 7 shows the optimal solution $H$ to the ACVRP in $\hat{G}$ given in Figure 6 corresponding to our example; it consists of two cycles:

- $H_{1}=\left(x_{D}, x_{a}, x_{b}, x_{D}\right)$ with associated cost $7+7+22+3 M=36+3 M$ (these arc costs will be deduced above when explaining the solution to the MCGRPTP in $G$ ) and the demand served by this cycle is $q_{a}+q_{b}=25+25=50$.

- $H_{2}=\left(x_{D}, x_{5 d c}, x_{5 g h}, x_{5 f h}, x_{5 f c}, x_{c_{2}}, x_{c_{1}}, x_{e_{2}}, x_{e_{1}}, x_{D}\right)$ with associated cost:

$15+0+0+0+0+0+4+0+12+4 M=31+4 M$ and the demand serviced by this cycle is $q_{c}+q_{e}+q_{v_{5}}=20+20+2+2+3+3=50$. Note that the total cost $\hat{c}(H)=67+7 M=36+31+(5+2) M$ since $m=5$ is the number of vertex subsets in $G^{*}$ (the depot not included) and $k=2$ is the number of cycles in the solution.

This optimal solution $H$ in $\hat{G}$ gives rise to the optimal solution $L$ in $G^{*}$ shown in Figure 8, with total cost $c^{*}(L)=\hat{c}(H)-7 M=67$, and that consists of two cycles: $C_{1}=\left(x_{D}, x_{a}, x_{b}, x_{D}\right)$ with cost 36 and $C_{2}=\left(x_{D}, x_{5 d c}, x_{c_{2}}, x_{e_{2}}, x_{D}\right)$ with cost 31. 


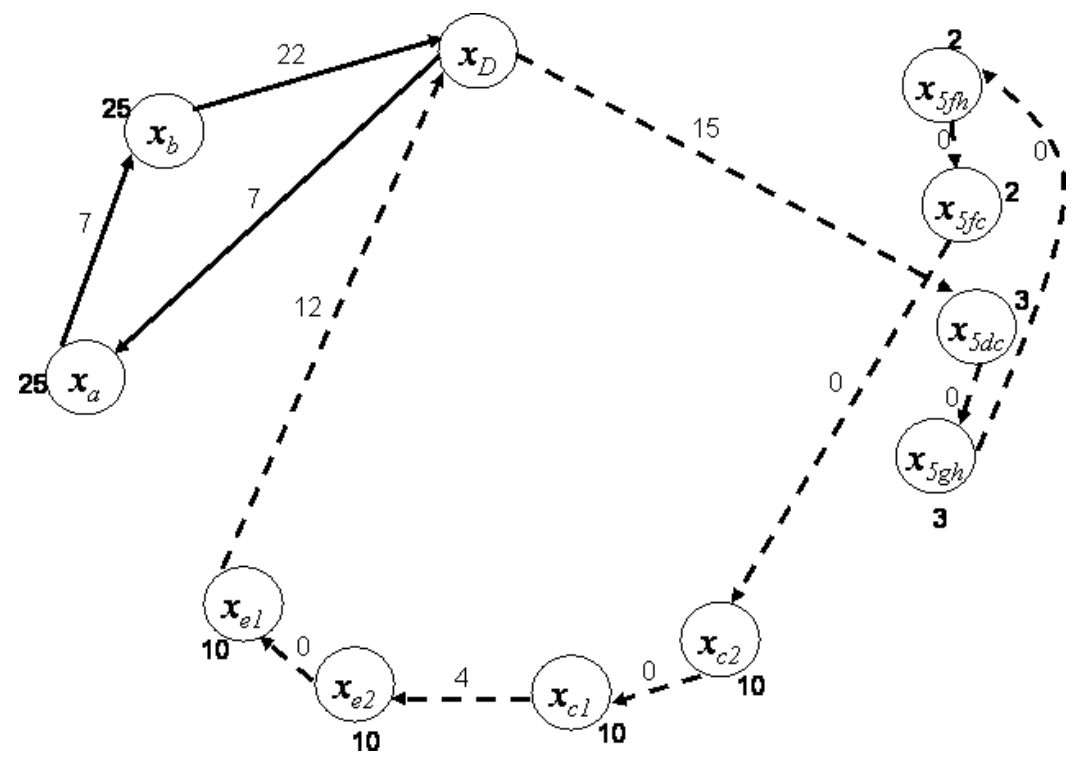

Figure 7. Optimal solution to the ACVRP in $\hat{G}$.

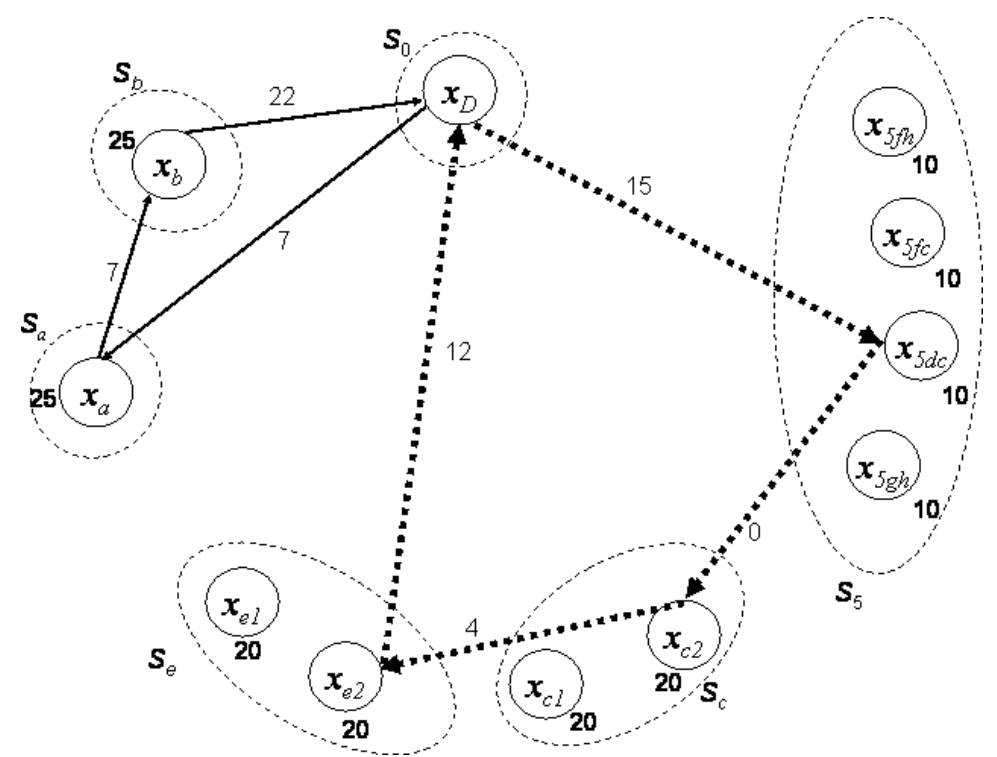

Figure 8. Optimal solution to the GVRP in $G^{*}$.

Let us find the optimal solution to the MCGRPTP in $G$ given by these cycles, according to the proof of Theorem 1.

From $C_{1}=\left(x_{D}, x_{a}, x_{b}, x_{D}\right)$ we have:

- $\left(x_{D}, x_{a}\right)$ corresponds to the s.f.c. $\left(1^{l^{a_{1}}}, 3^{a}\right)$ in $G^{\prime}:\left(1^{e}, 1^{l}\right)\left(1^{l}, 3\right)\left[\left(1^{l}, 3\right),(3,4)\right]$ with cost $0+\mathbf{0}+4+\mathbf{3}=7$ (in the addition, normal-font numbers indicate arc costs while bold numbers indicate turn penalties). See Figure 4 to follow the chains in $G^{\prime}$.

- $\left(x_{a}, x_{b}\right)$ corresponds to the $s . f . c .\left(4^{a}, 4^{b}\right)$ in $G^{\prime}:(3,4)[(3,4),(4,2)]$ with cost $4+3=7$. 
- $\left(x_{b}, x_{D}\right)$ corresponds to the s.f.c. $\left(2^{b}, 1^{e^{a_{1}}}\right)$ in $G^{\prime}:(4,2)\left(2,5_{f h}^{e}\right)\left(5_{f h}^{e} 5_{f h}^{l}\right)\left(5_{f h}^{l}, 4\right)\left(4,1^{e}\right)$ $\left[\left(4,1^{e}\right),\left(1^{e}, 1^{l}\right)\right]$ with cost $4+\mathbf{1}+4+\mathbf{1}+0+\mathbf{0}+5+\mathbf{1}+6+\mathbf{0}=22$.

Therefore, joining the chains we have the following feasible closed chain in $G^{\prime}$ $T_{1}^{\prime}=\left\{\left(1^{e}, 1^{l}\right),\left(1^{l}, 3\right),(3,4),(4,2),\left(2,5_{f h}^{e}\right),\left(5_{f h}^{e}, 5_{f h}^{l}\right),\left(5_{f h}^{l}, 4\right),\left(4,1^{e}\right),\left[\left(4,1^{e}\right),\left(1^{e}, 1^{l}\right)\right]\right\}$ with cost 36, and assuming the demands of $\operatorname{arcs}(3,4)$ and $(4,2)(a$ and $b)$, with a total demand of 50 .

From $T_{1}^{\prime}$, following again with the proof of Theorem 1, we construct the feasible closed chain with the same cost as $T_{1}^{\prime}$ in the original graph $G$,

$T_{1}=\{(1,3),(3,4),(4,2),(2,5)(5,4),(4,1),[(4,1),(1,3)]\}$ that also assumes the demands of arcs $a$ and $b(25+25)$.

Similarly, from $C_{2}=\left(x_{D}, x_{5 d c}, x_{c_{2}}, x_{e_{2}}, x_{D}\right)$ we have:

- $\left(x_{D}, x_{5 d c}\right)$ corresponds to the s.f.c. $\left(1^{l^{a_{1}}}, 5_{d c}^{e a_{5 d c}}\right)$ in $G^{\prime}$ :

$\left(1^{e}, 1^{l}\right)\left(1^{l}, 4\right)\left(4,5_{d c}^{e}\right)\left[\left(4,5_{d c}^{e}\right),\left(5_{d c}^{e}, 5_{d c}^{l}\right)\right]$ with cost $0+\mathbf{0}+6+\mathbf{3}+5+\mathbf{1}=15$.

- $\left(x_{5 d c}, x_{c_{2}}\right)$ corresponds to the s.f.c. $\left(5_{d c}^{l a_{5 d c}}, 5_{d c}^{l c_{2}}\right)$ in $G^{\prime}:\left(5_{d c}^{e}, 5_{d c}^{l}\right)\left[\left(5_{d c}^{e}, 5_{d c}^{l}\right),\left(5_{d c}^{l}, 6\right)\right]$ with cost $0+\mathbf{0}=0$.

- $\left(x_{c_{2}}, x_{e_{2}}\right)$ corresponds to the s.f.c. $\left(6^{c_{2}}, 6^{e_{2}}\right)$ in $G^{\prime}:\left(5_{d c}^{l}, 6\right)\left[\left(5_{d c}^{l}, 6\right),(6,4)\right]$ with cost $3+\mathbf{1}=4$.

- $\left(x_{e_{2}}, x_{D}\right)$ corresponds to the s.f.c. $\left(4^{e_{2}}, 1^{a^{a 1}}\right)$ in $G^{\prime}:(6,4)\left(4,1^{e}\right)\left[\left(4,1^{e}\right),\left(1^{e}, 1^{l}\right)\right]$ with cost $5+\mathbf{1}+6+\mathbf{0}=12$.

Therefore, joining the chains we have the following feasible tour in $G^{\prime}$,

$$
T_{2}^{\prime}=\left\{\left(1^{e}, 1^{l}\right),\left(1^{l}, 4\right),\left(4,5_{d c}^{e}\right),\left(5_{d c}^{e}, 5_{d c}^{l}\right),\left(5_{d c}^{l}, 6\right),(6,4),\left(4,1^{e}\right),\left[\left(4,1^{e}\right),\left(1^{e}, 1^{l}\right)\right]\right\} \text { with }
$$
cost 31 , and assuming the demands of $\operatorname{arcs}\left(5_{d c}^{e}, 5_{d c}^{l}\right),\left(5_{d c}^{l}, 6\right)$ and $(6,4)$, with a total demand of 50 .

From $T_{2}^{\prime}$, we construct the feasible closed chain with the same cost as $T_{2}^{\prime}$ in $G$,

$T_{2}=\{(1,4)(4,5)(5,6)(6,4)(4,1)[(4,1),(1,4)]\}$, that assumes the demands of vertex 5 belonging to $V_{R_{2}}$, of arc $(5,6)$ and of edge $(4,6)(10+20+20)$.

Figure 9 shows the optimal solution to the MCGRPTP in the original graph $G: T_{1}$ with solid bold line and $T_{2}$ with broken bold line. 


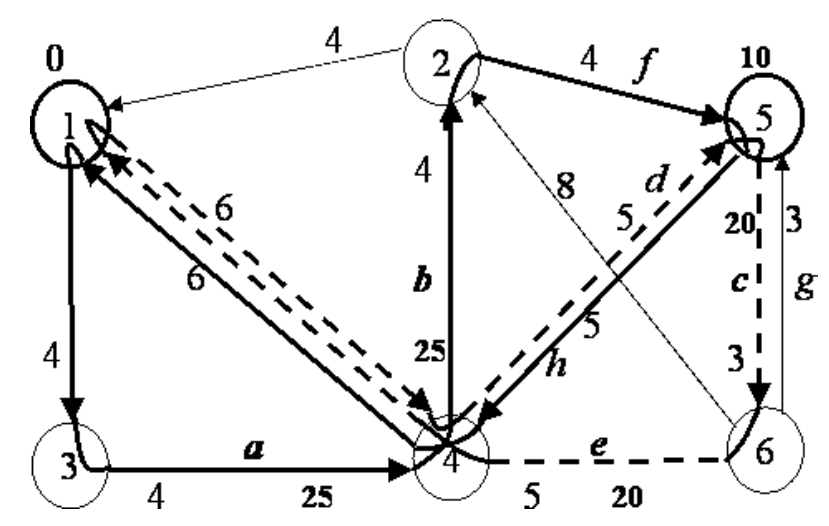

Figure 9. Optimal solution to the MCGRPTP in $G$.

\section{Computational experiments}

The aim of this section is to show that the transformation presented here can be considered as a good tool to solve MCGRPTP instances, at least heuristically due to the complexity of the problem. That is, if there exists any competitive procedure to solve the ACVRP, we can solve MCGRPTP instances within a reasonable running time. To do this, we first present a powerful heuristic algorithm for the ACVRP based on the memetic algorithm (MA) for the (symmetric) CVRP proposed by Nagata and Bräysy (2009). MA is a population-based heuristic search approach that combines evolutionary algorithm with local search algorithm. Although there are other heuristic approaches for the ACVRP, as those cited in the introduction, we selected the above mentioned MA because it is shown to be currently the most powerful heuristic method for the CVRP, and it can be applied to the ACVRP by a straightforward extension. Here the suggested MA has been tested on a set of 32 ACVRP instances by Pessoa et al (2007). We have also applied the MA to a set of 126 single vehicle instances by Soler et al (2008) because the optimal solutions are known to these instances. Finally, the MA has been applied to a set of 336 ACVRP instances with up to 623 vertices that come from the transformation of MCGRPTP instances.

\subsection{The MA for the ACVRP}

The main feature of the MA by Nagata and Bräysy (2009) is that the edge assembly crossover (EAX) operator generates offspring solutions by combining edges of two 
solutions selected as parents from the population. The generated offspring solutions may violate the capacity constraint. In this case, a subsequent local search-based repair procedure is used to restore the feasibility of the temporarily infeasible solutions. Moreover, a simple local search is applied to the obtained feasible solutions according to a standard MA procedure.

Note that the EAX was adapted to the ACVRP by defining it on the directed graph whereas the original EAX for the symmetric CVRP was defined on the undirected graph. The MA by Nagata and Bräysy minimized the total travel distance without putting any constraint on the number of vehicles and the number of vehicle was also a decision variable. However, according to the literature and therefore to the definition of the ACVRP given here, in the ACVRP the travel distance must be minimized with a given number of vehicles. So we have made a new version of the MA that minimizes the total travel distance for a fixed number of vehicles. The suggested MA has been implemented in $\mathrm{C}++$ and has been executed on a ADM Opteron $2.4 \mathrm{GHz}$ computer. For each instance, the MA has been executed five times.

\subsection{Results for the ACVRP instances}

We first analyze the efficiency of the two MA versions (fixed or variable number of vehicles) on a set of 32 ACVRP instances with known upper bounds that appear in the work by Pessoa et al (2007), which are variants of the 8 benchmark ACVRP instances given in http://or.ingce.unibo.it/research/cvrp-and-dcvrp. As far as we know, the work by Pessoa et al is the most recent paper with computational results on the ACVRP.

The results for the 32 ACVRP instances without constraint on the number of vehicles are presented in Table 1. The columns in the table list instance names (Instance), the capacity of the vehicles $(C)$, the number of vehicles and the total travel distance of the best-known upper bound solutions $(k$ and $U B$ ), the number of vehicles and the total travel distance of the best result in five runs (best- $k$ and best- $d$.) with our MA, the average number of vehicles and the average total travel distance in these five runs (ave- $k$ and ave- $d$.), and the average computation time in seconds for a run (Time). 
Table 1. Computational results on known ACVRP instances.

\begin{tabular}{|c|c|c|c|c|c|c|c|c|}
\hline Instance & $C$ & $k$ & Best UB & Best $-k$ & Best $-d$ & Ave $-k$ & Ave $-d$ & Time \\
\hline a034-14f & 150 & 14 & 4046 & 14 & 4046 & 14.0 & 4046.0 & 1.37 \\
\hline $\mathrm{a} 036-18 f$ & 150 & 18 & 5296 & 19 & 5224 & 19.0 & 5224.0 & 1.21 \\
\hline $\mathrm{a} 039-20 \mathrm{f}$ & 150 & 20 & 5903 & 20 & 5903 & 20.0 & 5903.0 & 1.47 \\
\hline $\mathrm{a} 045-18 \mathrm{f}$ & 150 & 18 & 6399 & 19 & 5564 & 19.0 & 5568.0 & 4.59 \\
\hline a048-16f & 150 & 16 & 4955 & 16 & 4955 & 16.6 & 4960.0 & 6.35 \\
\hline $\mathrm{a} 056-17 \mathrm{f}$ & 150 & 17 & 4998 & 17 & 4998 & 17.6 & 5020.0 & 8.82 \\
\hline a065-19f & 150 & 19 & 6014 & 20 & 5862 & 20.0 & 5862.0 & 12.52 \\
\hline $\mathrm{a} 071-17 \mathrm{f}$ & 150 & 17 & 5006 & 17 & 5006 & 17.0 & 5014.0 & 13.86 \\
\hline $\mathrm{a} 034-08 \mathrm{f}$ & 250 & 8 & 2672 & 8 & 2672 & 8.0 & 2672.0 & 1.94 \\
\hline $\mathrm{a} 036-10 \mathrm{f}$ & 250 & 10 & 3338 & 11 & 3294 & 11.0 & 3294.0 & 1.67 \\
\hline a039-12f & 250 & 12 & 3705 & 12 & 3705 & 12.0 & 3705.0 & 2.63 \\
\hline $\mathrm{a} 045-11 \mathrm{f}$ & 250 & 11 & 3544 & 11 & 3544 & 11.2 & 3555.6 & 5.41 \\
\hline $\mathrm{a} 048-10 \mathrm{f}$ & 250 & 10 & 3325 & 10 & 3325 & 10.0 & 3325.6 & 4.32 \\
\hline $\mathrm{a} 056-10 \mathrm{f}$ & 250 & 10 & 3263 & 10 & 3263 & 10.0 & 3263.0 & 7.46 \\
\hline $\mathrm{a} 065-12 \mathrm{f}$ & 250 & 12 & 3902 & 12 & 3902 & 12.0 & 3902.0 & 10.64 \\
\hline $\mathrm{a} 071-10 \mathrm{f}$ & 250 & 10 & 3486 & 10 & 3486 & 10.0 & 3486.6 & 12.73 \\
\hline a034-04f & 500 & 4 & 1773 & 4 & 1773 & 4.0 & 1773.0 & 1.76 \\
\hline $\mathrm{a} 036-05 \mathrm{f}$ & 500 & 5 & 2110 & 5 & 2110 & 5.0 & 2110.0 & 1.95 \\
\hline $\mathrm{a} 039-06 \mathrm{f}$ & 500 & 6 & 2289 & 6 & 2289 & 6.0 & 2289.0 & 2.00 \\
\hline $\mathrm{a} 045-06 \mathrm{f}$ & 500 & 6 & 2303 & 6 & 2303 & 6.0 & 2303.0 & 3.41 \\
\hline $\mathrm{a} 048-05 \mathrm{f}$ & 500 & 5 & 2283 & 5 & 2283 & 5.0 & 2283.0 & 3.26 \\
\hline $\mathrm{a} 056-05 \mathrm{f}$ & 500 & 5 & 2165 & 5 & 2165 & 5.0 & 2165.0 & 4.70 \\
\hline $\mathrm{a} 065-06 \mathrm{f}$ & 500 & 6 & 2567 & 6 & 2567 & 6.0 & 2568.0 & 7.82 \\
\hline $\mathrm{a} 071-05 \mathrm{f}$ & 500 & 5 & 2475 & 5 & 2457 & 5.0 & 2457.0 & 7.62 \\
\hline $\mathrm{a} 034-02 \mathrm{f}$ & 1000 & 2 & 1406 & 2 & 1406 & 2.0 & 1406.0 & 1.31 \\
\hline a036-03f & 1000 & 3 & 1644 & 3 & 1644 & 3.0 & 1644.0 & 1.13 \\
\hline a039-03f & 1000 & 3 & 1654 & 3 & 1654 & 3.0 & 1654.0 & 1.17 \\
\hline a045-03f & 1000 & 3 & 1740 & 3 & 1740 & 3.0 & 1740.0 & 1.97 \\
\hline $\mathrm{a} 048-03 \mathrm{f}$ & 1000 & 3 & 1891 & 3 & 1891 & 3.0 & 1891.0 & 1.69 \\
\hline a056-03f & 1000 & 3 & 1739 & 3 & 1739 & 3.0 & 1739.0 & 3.23 \\
\hline a065-03f & 1000 & 3 & 1974 & 3 & 1974 & 3.0 & 1974.0 & 4.17 \\
\hline a071-03f & 1000 & 3 & 2054 & 3 & 2054 & 3.0 & 2054.0 & 4.24 \\
\hline
\end{tabular}


We can see that in four instances out of the 32 instances the MA has improved the best known upper bound by using one more vehicle, and in the other 28 instances the solution given by the MA coincides with the best known solution both in the total travel distance and in the number of vehicles.

We also have run the MA with fixing a priori the number of vehicles equal to the one given in the best known solution (see third column in Table 1). In this case, in all of the 32 instances the total travel distance obtained with the MA coincides with the best known upper bound, with running time similar to the one given in Table 1. Therefore, the table corresponding to these results has been omitted.

In Table 2 we show the results to the set of 126 single vehicle instances given by Soler et al (2008) for which the optimal solution is known. In this table, results are averaged over selected groups of the instances where the 126 instances are partitioned into 24 groups according to features of the instances. The columns in the table lists the number of instances in each groups (Ins), the average number of required arcs in the subset $(A R A)$, the number of (required) edges in all the instances in the subset $(|E|)$, the average number of required vertices in the subset $(A R V)$, the average number of vertices in the asymmetric TSP instances obtained from the original instances $\left(A V_{A T S P}\right)$, the average time in seconds to obtain the optimal solution with the exact procedure $(A T O)$, the average time in seconds to obtain the heuristic solution with the MA $(A T M A)$, the number of instances optimally solved with the MA in the subset $(O P T)$, and the average deviation of the MA solutions in the subset $(A D E V)$. By deviation we mean $(U B-L B) / L B$. 
Table 2. Computational results on known single vehicle instances.

\begin{tabular}{cccccccccc}
\hline Group & Ins & $A R A$ & $|E|$ & $A R V$ & $A V_{A T S P}$ & $A T O$ & $A T M A$ & OPT & $A D E V$ \\
\hline 1 & 6 & 51 & 0 & 6 & 82 & 14.48 & 5,76 & 6 & 0 \\
2 & 4 & 84 & 0 & 8 & 127 & 65.04 & 11,04 & 4 & 0 \\
3 & 6 & 108 & 0 & 8 & 149 & 392,81 & 16,20 & 6 & 0 \\
4 & 5 & 125 & 0 & 12 & 195 & 348,34 & 27,89 & 4 & 0,00010 \\
5 & 7 & 145 & 0 & 14 & 212 & 932,43 & 26,87 & 6 & 0,00050 \\
6 & 6 & 164 & 0 & 16 & 248 & 1269,71 & 41,29 & 5 & 0,00025 \\
7 & 5 & 54 & 0 & 3 & 86 & 42.65 & 7,53 & 4 & 0,00078 \\
8 & 4 & 84 & 10 & 8 & 137 & 478,8 & 12,73 & 4 & 0 \\
9 & 7 & 107 & 10 & 9 & 175 & 490,38 & 21,33 & 6 & 0,00071 \\
10 & 6 & 126 & 10 & 11 & 201 & 819,55 & 29,76 & 1 & 0,00059 \\
11 & 5 & 141 & 10 & 13 & 231 & 1273,89 & 41,44 & 4 & 0,00052 \\
12 & 6 & 156 & 10 & 14 & 245 & 1037,18 & 50,19 & 5 & 0,00007 \\
13 & 6 & 172 & 10 & 15 & 265 & 3211,59 & 61,19 & 2 & 0,00093 \\
14 & 5 & 61 & 10 & 3 & 119 & 139,92 & 7,18 & 5 & 0 \\
15 & 6 & 105 & 20 & 5 & 167 & 1464,62 & 21,52 & 3 & 0,00049 \\
16 & 4 & 126 & 20 & 9 & 208 & 516,69 & 40,62 & 1 & 0,00141 \\
17 & 6 & 142 & 20 & 11 & 240 & 3095,38 & 43,53 & 2 & 0,00142 \\
18 & 4 & 156 & 20 & 9 & 236 & 3539,15 & 42,90 & 3 & 0,00089 \\
19 & 3 & 168 & 20 & 12 & 236 & 3646,25 & 66,26 & 1 & 0,00130 \\
20 & 4 & 58 & 20 & 3 & 129 & 846,88 & 16,77 & 2 & 0,00088 \\
21 & 6 & 100 & 20 & 5 & 187 & 1218,35 & 22,66 & 4 & 0,00029 \\
22 & 6 & 65 & 30 & 3 & 156 & 2437,78 & 22,63 & 3 & 0,00034 \\
23 & 6 & 113 & 30 & 6 & 218 & 2931 & 37,87 & 1 & 0,00087 \\
24 & 3 & 140 & 30 & 6 & 191 & $>2 h$. & 64,47 & 3 & 0 \\
\hline & & & & & & & & &
\end{tabular}

We can see from Table 2 that the results obtained with the MA are very good. The MA was able to optimally solve 86 instances out of the 126 instances, with $0.05 \%$ average deviation.

\subsection{Solving MCGRPTP instances with the MA}

We applied the MA to solve also three sets of random MCGRPTP instances with up to 700 arcs, 160 (required) edges, 160 vertices, 225 required arcs, and 28 required 
vertices, which are transformed into ACVRP instances with up to 623 vertices. As far as we know, the largest ACVRP instance described in the literature until now has 300 vertices.

Next we describe the data generation procedure for each set.

The first set was generated from the 128 single vehicle instances given by Soler et al (2008), and we obtain two MCGRPTP instance sets (one with vehicle capacity 1000 and the other with vehicle capacity 2000) as follows:

We choose the depot node as the first required vertex in the numerical order. In this node all turns are changed to be allowed with zero cost. Each required arc, required edge and required vertex will have a randomly generated integer demand in range $[13,120],[7,60]$, and $[50,120]$ respectively. Note that when we transform one of these MCGRPTP instances into an ACVRP instance, if an original required vertex $v$ has demand $d_{v}$ and it gives rise to a vertex subset in the ACVRP with $t$ vertices, let $r$ be the largest integer positive number such that $d_{v}=r t+c$, with $c \geq 0$, then $t-1$ vertices in this subset will have demand $r$, and the last one will have demand $r+c$. These 256 generated MCGRPTP instances act as a basis for ACVRP instances with number of vertices in the interval $[61,290]$.

The second set contains 32 MCGRPTP instances generated from 16 of the biggest single vehicle instances by Corberán et al (2002). Each original instance is first transformed into an MGRP with turn penalties instance with the procedure explained in Soler et al (2008) and then transformed into two MCGRPTP instances (one with capacity 1000 and the other with capacity 2000) with the procedure given above for the first set. These 32 instances form the ACVRP instances with number of vertices in the interval $[235,406]$.

Finally, the third set contains 48 instances that have been obtained from 24 new large single vehicle instances randomly generated with the same instance generator used by Corberán et al (2002). These 48 MCGRPTP instances are transformed into ACVRP instances with the number of vertices in the interval $[382,623]$.

In these three sets we have used the MA version with variable number of vehicles 
in order to obtain best upper bounds with respect to the total travel distance. The appendix shows a table containing all data and results corresponding to each individual MCGRPTP instances. In this table, each instance is named as Ixxy, where xx indicates the subset to which the instance belongs and y indicates the number of the instance inside that subset. The first 24 subsets correspond to the first set of (128) instances, subsets 25 to 27 correspond to the second set of (16) instances and subsets 28 to 31 correspond to the third set of (24) instances. The columns in the table list the following data: the number of vertices in the ACVRP instance obtained from the corresponding MCGRPTP instance $\left(\left|V_{A C V R P}\right|\right)$, the total demand in the ACVRP instance (T.D.), and for $i \in\{1,2\}$, the total travel distance of the best result in five runs obtained for a vehicle capacity of $C_{i}=i \cdot 1000$ units $\left(d_{i}\right)$, the number of vehicles corresponding to this best result $\left(K_{i}\right)$ and the average computing time in seconds for a run corresponding to the capacity $C_{i}\left(\right.$ Time $\left._{i}\right)$. Note that $d_{i}$ is the total distance in the original MCGRPTP instance, not in the auxiliary ACVRP instance.

The MA was able to find feasible solutions for all 336 instances including the largesize instances with up to 600 vertices, within a reasonable computation time, as reported in the appendix. The computation times vary from a few seconds to more than one hour depending on the problem size. We consider the reported computing times reasonable, given the size and complexity of the considered ACVRP instances.

Based on these results, one can conclude that at least medium-size real-world MCGRPTPs can be solved by a state-of-the-art heuristic method for the ACVRP through their transformation into an ACVRP as explained here. By the way, we have generated a large number of instances to the, until now, limited set of ACVRP benchmarck instances. Of course these new instances will be available to any researcher interested on them.

\section{Conclusions}

In this paper we have studied a generalization of the MCGRP including turn penalties and forbidden turns. Through an intermediate transformation into a GVRP, we have provided a procedure to transform it into an ACVRP. Then, at least from a theoretical 
point of view, this generalization can be solved both optimally and heuristically with existing algorithms. We have also introduced a set of new benchmark problems and adapted a recent and powerful memetic algorithm to ACVRP. The experimental results show an average deviation equal to $0.05 \%$ for instances with known optimal solution and that large-size problems can be solved with the memetic algorithm.

We are convinced that research on turn penalties will increase and be of important value in the future to reduce the gap between theoretical research and real-life applications. We hope that the theoretical and experimental results presented here can be used in the future as ideas or tools to test the efficiency of specific procedures to solve capacitated routing problems with turn penalties.

\section{Acknowledgements}

This work has been partially supported by the Ministerio de Educación y Ciencia of Spain (project TIN2008-06441-C02-01). 


\section{Appendix}

\begin{tabular}{|c|c|c|c|c|c|c|c|c|c|c|c|}
\hline Inst. & $|V|-|A|-|E|$ & $\left|A_{R}\right|$ & $\left|V_{R}\right|$ & $\left|V_{A C V R P}\right|$ & T.D. & $d_{1}$ & $K_{1}$ & Time $_{1}$ & $d_{2}$ & $K_{2}$ & Time $_{2}$ \\
\hline $\mathrm{I} 11$ & 40-110-0 & 44 & 3 & 61 & 3819 & 4255 & 4 & 13.11 & 3966 & 2 & 4.42 \\
\hline $\mathrm{I} 12$ & $60-140-0$ & 56 & 5 & 75 & 6462 & 6848 & 5 & 25.63 & 6145 & 3 & 17.9 \\
\hline $\mathrm{I} 13$ & $40-90-0$ & 36 & 3 & 46 & 3009 & 4103 & 4 & 11.04 & 3547 & 2 & 19.67 \\
\hline I14 & $80-170-0$ & 68 & 6 & 92 & 5415 & 7578 & 6 & 25.15 & 6806 & 3 & 20.9 \\
\hline $\mathrm{I} 15$ & $40-90-0$ & 36 & 6 & 54 & 3142 & 4126 & 4 & 7.56 & 3625 & 2 & 8.38 \\
\hline $\mathrm{I} 16$ & $80-170-0$ & 68 & 11 & 118 & 5772 & 7651 & 6 & 65.18 & 6798 & 3 & 37.18 \\
\hline $\mathrm{I} 21$ & $80-200-0$ & 80 & 7 & 107 & 6936 & 7464 & 7 & 53.7 & 6962 & 3 & 35.13 \\
\hline $\mathrm{I} 22$ & $100-220-0$ & 88 & 7 & 121 & 7067 & 10152 & 8 & 41.1 & 8999 & 4 & 28.14 \\
\hline $\mathrm{I} 23$ & $80-200-0$ & 80 & 4 & 95 & 5815 & 7300 & 6 & 53.97 & 7026 & 4 & 28.3 \\
\hline $\mathrm{I} 24$ & $100-220-0$ & 88 & 14 & 158 & 8907 & 10643 & 9 & 79.62 & 9439 & 5 & 54.04 \\
\hline I31 & $100-260-0$ & 104 & 3 & 112 & 6704 & 11831 & 7 & 63.81 & 11272 & 4 & 24.74 \\
\hline I32 & $120-260-0$ & 104 & 6 & 127 & 8055 & 13666 & 11 & 256.67 & 12364 & 6 & 112.48 \\
\hline I33 & $120-290-0$ & 116 & 7 & 147 & 8946 & 13493 & 9 & 228.25 & 12332 & 5 & 59.84 \\
\hline I34 & $100-260-0$ & 104 & 6 & 136 & 8154 & 12352 & 9 & 62.52 & 11466 & 5 & 47.86 \\
\hline I35 & $120-260-0$ & 104 & 11 & 147 & 8387 & 12264 & 9 & 43 & 11130 & 5 & 41.33 \\
\hline I36 & $120-290-0$ & 116 & 14 & 195 & 10603 & 14253 & 11 & 248.94 & 12728 & 6 & 102.05 \\
\hline I41 & $140-300-0$ & 120 & 8 & 151 & 9108 & 15107 & 10 & 93.41 & 13086 & 5 & 69.21 \\
\hline $\mathrm{I} 42$ & $140-330-0$ & 132 & 12 & 216 & 10775 & 16681 & 11 & 320.16 & 15176 & 6 & 114.81 \\
\hline $\mathrm{I} 43$ & $140-330-0$ & 132 & 6 & 164 & 10232 & 16542 & 11 & 139.86 & 15126 & 6 & 65.39 \\
\hline $\mathrm{I} 44$ & $140-300-0$ & 120 & 20 & 220 & 11314 & 16130 & 12 & 214.2 & 13732 & 6 & 156.12 \\
\hline $\mathrm{I} 45$ & $140-300-0$ & 120 & 14 & 185 & 9746 & 15101 & 10 & 204.5 & 13184 & 5 & 122.74 \\
\hline $\mathrm{I} 51$ & $160-340-0$ & 136 & 8 & 166 & 10460 & 14698 & 11 & 156.37 & 13806 & 7 & 224.51 \\
\hline $\mathrm{I} 52$ & $160-340-0$ & 136 & 19 & 219 & 8387 & 15947 & 14 & 284.86 & 13405 & 6 & 69 \\
\hline I53 & $160-340-0$ & 136 & 13 & 191 & 11192 & 14987 & 12 & 183.21 & 13413 & 6 & 117.01 \\
\hline I54 & $180-380-0$ & 152 & 8 & 183 & 10750 & 18322 & 11 & 219.97 & 16710 & 6 & 92.44 \\
\hline $\mathrm{I} 55$ & $160-370-0$ & 148 & 14 & 218 & 12729 & 17818 & 13 & 387.24 & 15959 & 7 & 151.67 \\
\hline $\mathrm{I} 56$ & $180-380-0$ & 152 & 21 & 250 & 13252 & 19431 & 14 & 422.96 & 17000 & 7 & 236.26 \\
\hline $\mathrm{I} 57$ & $180-380-0$ & 152 & 15 & 217 & 12690 & 18806 & 13 & 369.88 & 16687 & 7 & 117.98 \\
\hline I61 & $200-420-0$ & 168 & 10 & 210 & 12868 & 21039 & 13 & 431.41 & 18289 & 7 & 131.69 \\
\hline I62 & $180-400-0$ & 160 & 8 & 193 & 12364 & 19634 & 13 & 227.68 & 17122 & 7 & 91.19 \\
\hline I63 & $200-420-0$ & 168 & 17 & 289 & 15961 & 25569 & 16 & 1608.23 & 19197 & 9 & 348.06 \\
\hline I64 & $180-400-0$ & 160 & 20 & 255 & 14227 & 20806 & 15 & 522.98 & 17808 & 8 & 214.22 \\
\hline I65 & $180-400-0$ & 160 & 14 & 217 & 13445 & 20295 & 14 & 392.63 & 17340 & 7 & 180.87 \\
\hline I66 & $200-420-0$ & 168 & 18 & 254 & 14903 & 22681 & 15 & 669.9 & 18661 & 8 & 235.08 \\
\hline I71 & $40-110-10$ & 44 & 1 & 65 & 3612 & 4830 & 4 & 12.93 & 4528 & 2 & 11.29 \\
\hline $\mathrm{I} 72$ & $40-90-10$ & 36 & 3 & 67 & 3501 & 4353 & 4 & 14.94 & 4151 & 2 & 16.83 \\
\hline I73 & $60-140-10$ & 56 & 2 & 81 & 4290 & 6101 & 5 & 16.09 & 5808 & 3 & 16.85 \\
\hline I74 & $60-160-10$ & 64 & 2 & 89 & 5011 & 7201 & 6 & 28.32 & 6859 & 3 & 30.66 \\
\hline $\mathrm{I} 75$ & $80-170-10$ & 68 & 4 & 107 & 6462 & 7924 & 7 & 45.76 & 7248 & 4 & 31.74 \\
\hline I81 & $80-200-10$ & 80 & 4 & 113 & 6703 & 7403 & 7 & 62.7 & 6993 & 4 & 30.44 \\
\hline I82 & $100-220-10$ & 88 & 5 & 113 & 6824 & 7409 & 7 & 86.16 & 6993 & 4 & 34.07 \\
\hline I 83 & $80-200-10$ & 80 & 7 & 133 & 7279 & 7654 & 8 & 51.37 & 7034 & 4 & 47.82 \\
\hline I84 & $100-220-10$ & 88 & 10 & 184 & 9826 & 9568 & 9 & 109.2 & 8820 & 5 & 47.64 \\
\hline
\end{tabular}




\begin{tabular}{|c|c|c|c|c|c|c|c|c|c|c|c|}
\hline Inst. & $|V|-|A|-|E|$ & $\left|A_{R}\right|$ & $\left|V_{R}\right|$ & $\left|V_{A C V R P}\right|$ & T.D. & $d_{1}$ & $K_{1}$ & Time $_{1}$ & $d_{2}$ & $K_{2}$ & $\mathrm{Time}_{2}$ \\
\hline I91 & $120-260-10$ & 104 & 6 & 149 & 8587 & 13401 & 11 & 157.11 & 12181 & 6 & 78.18 \\
\hline I92 & $100-260-10$ & 104 & 5 & 157 & 8386 & 12508 & 9 & 209.1 & 11584 & 5 & 81 \\
\hline I93 & $120-290-10$ & 116 & 5 & 158 & 8700 & 12457 & 9 & 145.08 & 11757 & 5 & 47.07 \\
\hline I94 & $120-260-10$ & 104 & 16 & 204 & 10556 & 12734 & 9 & 111.9 & 11894 & 5 & 60.81 \\
\hline I95 & $100-260-10$ & 104 & 10 & 184 & 9826 & 13780 & 10 & 248.3 & 12055 & 5 & 129.32 \\
\hline I96 & $120-290-10$ & 116 & 10 & 183 & 9539 & 12771 & 10 & 182.84 & 11934 & 5 & 106.03 \\
\hline I97 & $120-260-10$ & 104 & 11 & 177 & 10132 & 12703 & 9 & 95.41 & 11877 & 5 & 61.5 \\
\hline I101 & $140-300-10$ & 120 & 6 & 169 & 9675 & 14174 & 10 & 190.19 & 12609 & 5 & 121.77 \\
\hline I102 & $140-330-10$ & 132 & 6 & 179 & 10520 & 16205 & 11 & 195.82 & 15124 & 6 & 64.7 \\
\hline I103 & $140-330-10$ & 132 & 11 & 203 & 11670 & 16628 & 12 & 330.09 & 15468 & 6 & 172.42 \\
\hline I104 & $140-300-10$ & 120 & 16 & 215 & 11522 & 15189 & 12 & 314.17 & 13089 & 6 & 169.71 \\
\hline I105 & $140-330-10$ & 132 & 15 & 243 & 13154 & 17056 & 14 & 389.77 & 15618 & 7 & 177.68 \\
\hline I106 & $140-300-10$ & 120 & 11 & 193 & 10832 & 14880 & 11 & 274.03 & 12822 & 6 & 102.74 \\
\hline I111 & $160-340-10$ & 136 & 9 & 193 & 11457 & 16445 & 12 & 273.48 & 14640 & 6 & 172.66 \\
\hline I112 & $160-340-10$ & 136 & 23 & 275 & 13274 & 17141 & 14 & 484.04 & 14918 & 7 & 330.28 \\
\hline I113 & $160-340-10$ & 136 & 16 & 230 & 12651 & 16616 & 13 & 403.95 & 14690 & 7 & 152.03 \\
\hline I114 & $160-370-10$ & 148 & 6 & 195 & 12448 & 18102 & 13 & 247.19 & 16335 & 7 & 113.22 \\
\hline I115 & $160-370-10$ & 148 & 12 & 230 & 13856 & 19240 & 14 & 586 & 16446 & 7 & 323.42 \\
\hline I121 & $180-380-10$ & 152 & 8 & 203 & 11981 & 20136 & 12 & 782.32 & 17250 & 8 & 397.97 \\
\hline I122 & $180-380-10$ & 152 & 20 & 277 & 15076 & 20133 & 16 & 530.39 & 16547 & 6 & 406.85 \\
\hline I123 & $180-380-10$ & 152 & 14 & 234 & 12662 & 18966 & 13 & 500.68 & 16671 & 7 & 211.59 \\
\hline I124 & $180-400-10$ & 160 & 13 & 241 & 13650 & 18237 & 14 & 411.86 & 16348 & 7 & 265.88 \\
\hline $\mathrm{I} 125$ & $180-400-10$ & 160 & 8 & 219 & 12867 & 18404 & 13 & 431.07 & 16303 & 7 & 169.24 \\
\hline I126 & $180-400-10$ & 160 & 19 & 276 & 15023 & 18594 & 16 & 415.15 & 16647 & 8 & 308.28 \\
\hline I131 & $200-420-10$ & 168 & 8 & 234 & 14073 & 20264 & 13 & 454.48 & 18009 & 7 & 153.91 \\
\hline I132 & $200-440-10$ & 176 & 8 & 223 & 12780 & 21636 & 15 & 308.71 & 19289 & 8 & 140.3 \\
\hline I133 & $200-420-10$ & 168 & 22 & 288 & 15931 & 23868 & 16 & 1173.59 & 19076 & 8 & 661.17 \\
\hline I134 & $200-440-10$ & 176 & 21 & 290 & 15052 & 21369 & 16 & 557.87 & 18686 & 8 & 343.22 \\
\hline I135 & $200-420-10$ & 168 & 15 & 257 & 14872 & 21877 & 15 & 689.08 & 18479 & 8 & 278.81 \\
\hline I136 & $200-440-10$ & 176 & 15 & 275 & 15877 & 24410 & 16 & 790.1 & 20049 & 8 & 522.07 \\
\hline I141 & $60-90-20$ & 36 & 1 & 77 & 3699 & 4084 & 4 & 20.51 & 3833 & 2 & 21.02 \\
\hline I142 & $60-160-20$ & 64 & 5 & 115 & 5659 & 8403 & 7 & 52.74 & 7629 & 4 & 30.23 \\
\hline I143 & $60-140-20$ & 56 & 5 & 129 & 6107 & 7284 & 6 & 64.19 & 6522 & 3 & 42.57 \\
\hline I144 & $80-170-20$ & 68 & 5 & 125 & 6565 & 8181 & 7 & 82.09 & 7743 & 4 & 52.77 \\
\hline I145 & $80-200-20$ & 80 & 1 & 121 & 6542 & 7986 & 7 & 58.58 & 7620 & 4 & 49.68 \\
\hline I151 & $100-220-20$ & 88 & 4 & 141 & 8387 & 10444 & 8 & 195.27 & 9800 & 4 & 106.95 \\
\hline I152 & $120-260-20$ & 104 & 4 & 157 & 8391 & 12665 & 9 & 102.45 & 11959 & 5 & 76.46 \\
\hline I153 & $120-290-20$ & 116 & 3 & 169 & 10033 & 13550 & 11 & 156.71 & 12303 & 6 & 76.88 \\
\hline I154 & $100-260-20$ & 104 & 3 & 153 & 8387 & 13614 & 11 & 189.79 & 12502 & 6 & 98.08 \\
\hline I155 & $120-260-20$ & 104 & 7 & 171 & 9857 & 13125 & 10 & 299.63 & 12032 & 5 & 159.41 \\
\hline I156 & $120-290-20$ & 116 & 6 & 183 & 9777 & 13596 & 10 & 257.16 & 12317 & 5 & 172.63 \\
\hline I161 & $140-300-20$ & 120 & 6 & 184 & 10131 & 15037 & 11 & 133.85 & 13473 & 6 & 77.48 \\
\hline I162 & $140-330-20$ & 132 & 6 & 196 & 10861 & 16840 & 11 & 304.8 & 14957 & 6 & 137.01 \\
\hline I163 & $140-300-20$ & 120 & 11 & 208 & 11277 & 15554 & 12 & 239.37 & 13585 & 6 & 136.5 \\
\hline I164 & $140-330-20$ & 132 & 11 & 235 & 12807 & 17620 & 13 & 497.14 & 15329 & 7 & 175.13 \\
\hline
\end{tabular}




\begin{tabular}{|c|c|c|c|c|c|c|c|c|c|c|c|}
\hline Inst. & $|V|-|A|-|E|$ & $\left|A_{R}\right|$ & $\left|V_{R}\right|$ & $\left|V_{A C V R P}\right|$ & T.D. & $d_{1}$ & $K_{1}$ & Time $_{1}$ & $d_{2}$ & $K_{2}$ & $\mathrm{Time}_{2}$ \\
\hline I171 & $160-340-20$ & 136 & 16 & 249 & 13059 & 16078 & 14 & 418.03 & 14551 & 7 & 261.08 \\
\hline $\mathrm{I} 172$ & $160-340-20$ & 136 & 6 & 197 & 11250 & 15202 & 12 & 251 & 14189 & 6 & 154.1 \\
\hline I173 & $160-370-20$ & 148 & 6 & 214 & 12080 & 18298 & 13 & 237.53 & 16272 & 7 & 144.29 \\
\hline I174 & $160-340-20$ & 136 & 11 & 224 & 11824 & 15763 & 12 & 388.2 & 14244 & 6 & 325.71 \\
\hline I175 & $160-370-20$ & 148 & 11 & 245 & 13134 & 18765 & 14 & 355.11 & 16396 & 7 & 248.01 \\
\hline I176 & $160-370-20$ & 148 & 16 & 288 & 14216 & 19257 & 15 & 500.41 & 16827 & 8 & 247.63 \\
\hline I181 & $180-380-20$ & 152 & 5 & 212 & 11643 & 18628 & 12 & 332.17 & 16663 & 6 & 246.78 \\
\hline I182 & $180-380-20$ & 152 & 9 & 230 & 12888 & 19989 & 13 & 756.61 & 17339 & 7 & 231.97 \\
\hline I183 & $180-400-20$ & 160 & 7 & 229 & 13028 & 18463 & 14 & 303.72 & 16648 & 7 & 190.11 \\
\hline I184 & $180-400-20$ & 160 & 14 & 267 & 13991 & 18851 & 15 & 1343.62 & 18193 & 7 & 914.38 \\
\hline I191 & $200-420-20$ & 168 & 7 & 237 & 13896 & 22059 & 14 & 703.29 & 19778 & 8 & 802.64 \\
\hline I192 & $200-420-20$ & 168 & 17 & 289 & 15961 & 25569.00 & 16 & 1608.23 & 18782 & 7 & 406.29 \\
\hline I193 & $200-420-20$ & 168 & 12 & 267 & 14877 & 23137 & 15 & 907.11 & 19184 & 8 & 251.88 \\
\hline I 201 & $40-110-30$ & 44 & 1 & 105 & 5266 & 6069 & 6 & 40.75 & 5444 & 3 & 38.24 \\
\hline I 202 & $60-140-30$ & 56 & 2 & 123 & 5777 & 6705 & 6 & 96.64 & 6359 & 3 & 54.63 \\
\hline I 203 & 60-160-30 & 64 & 2 & 129 & 6846 & 8571 & 7 & 154.47 & 7915 & 4 & 80.08 \\
\hline I 204 & $80-170-30$ & 68 & 5 & 144 & 7021 & 8487 & 8 & 66.81 & 7896 & 4 & 74.44 \\
\hline I 211 & $80-200-30$ & 80 & 3 & 149 & 7597 & 8704 & 8 & 131.29 & 7943 & 4 & 88.27 \\
\hline $\mathrm{I} 212$ & $80-200-30$ & 80 & 6 & 163 & 7744 & 8818 & 8 & 198.14 & 8056 & 4 & 103.63 \\
\hline I 213 & $100-260-30$ & 104 & 1 & 192 & 10061 & 12822 & 11 & 284.98 & 11984 & 6 & 143.23 \\
\hline I 214 & $120-260-30$ & 104 & 5 & 183 & 8387 & 12670 & 10 & 161.83 & 11516 & 5 & 122.43 \\
\hline $\mathrm{I} 215$ & $120-290-30$ & 116 & 6 & 199 & 10056 & 13642 & 11 & 166.43 & 12382 & 6 & 116.95 \\
\hline I 216 & $120-290-30$ & 116 & 11 & 233 & 12343 & 14384 & 13 & 356.58 & 12711 & 7 & 152.71 \\
\hline $\mathrm{I} 221$ & $40-90-40$ & 36 & 1 & 117 & 5416 & 5321 & 6 & 59.06 & 4962 & 3 & 56.52 \\
\hline $\mathrm{I} 222$ & $60-140-40$ & 56 & 3 & 147 & 7012 & 7742 & 8 & 88.71 & 7093 & 4 & 81.97 \\
\hline $\mathrm{I} 223$ & $60-160-40$ & 64 & 2 & 149 & 7524 & 8892 & 8 & 150.32 & 8225 & 4 & 90.57 \\
\hline $\mathrm{I} 224$ & $80-170-40$ & 68 & 1 & 149 & 7651 & 8818 & 8 & 129.22 & 8134 & 4 & 82.15 \\
\hline I 225 & $100-220-40$ & 88 & 5 & 214 & 10793 & 11285 & 11 & 368.81 & 10027 & 6 & 160.24 \\
\hline I 226 & $80-200-40$ & 80 & 2 & 165 & 7986 & 9410 & 8 & 509.77 & 8253 & 4 & 275.54 \\
\hline I 231 & $120-260-40$ & 104 & 3 & 207 & 10567 & 14740 & 11 & 403.89 & 13088 & 6 & 145.29 \\
\hline I 232 & $120-290-40$ & 116 & 3 & 207 & 10678 & 14281 & 11 & 430.29 & 13110 & 6 & 150.35 \\
\hline $\mathrm{I} 233$ & $120-290-40$ & 116 & 6 & 221 & 11151 & 14642 & 12 & 369.99 & 13282 & 6 & 178.76 \\
\hline I 234 & $100-260-40$ & 104 & 5 & 208 & 10307 & 12485 & 9 & 88.39 & 11297 & 5 & 50.78 \\
\hline I 235 & $140-300-40$ & 120 & 5 & 216 & 11258 & 15690 & 12 & 327.21 & 14373 & 6 & 202.45 \\
\hline I 236 & $140-300-40$ & 120 & 10 & 242 & 7021 & 16012 & 13 & 460.42 & 14576 & 7 & 207.78 \\
\hline $\mathrm{I} 241$ & $140-330-40$ & 132 & 5 & 231 & 11865 & 17444 & 12 & 606.84 & 15789 & 6 & 372.98 \\
\hline $\mathrm{I} 242$ & $160-340-40$ & 136 & 6 & 241 & 12797 & 17189 & 13 & 687.33 & 15339 & 7 & 207.94 \\
\hline I 243 & $160-370-40$ & 148 & 5 & 249 & 13426 & 18010 & 14 & 552.3 & 16245 & 7 & 365.67 \\
\hline I 244 & $160-340-40$ & 136 & 12 & 271 & 13973 & 20563 & 14 & 1586.52 & 16225 & 7 & 904.7 \\
\hline I 245 & $160-370-40$ & 148 & 10 & 275 & 14247 & 18365 & 15 & 566.35 & 16602 & 8 & 239.05 \\
\hline $\mathrm{I} 251$ & $120-260-60$ & 104 & 3 & 235 & 10952 & 16098 & 11 & 836.73 & 13467 & 6 & 287.68 \\
\hline $\mathrm{I} 252$ & $140-330-60$ & 132 & 7 & 283 & 13898 & 18238 & 14 & 1108.62 & 15406 & 7 & 799.45 \\
\hline I 253 & $160-370-60$ & 148 & 6 & 293 & 14768 & 20488 & 15 & 1189.57 & 18175 & 8 & 476.2 \\
\hline I 254 & $180-400-60$ & 160 & 8 & 315 & 16339 & 21517 & 17 & 1184.31 & 19329 & 9 & 522.9 \\
\hline $\mathrm{I} 256$ & $200-420-60$ & 168 & 17 & 361 & 19015 & 24841 & 20 & 1212.79 & 21615 & 10 & 1023.6 \\
\hline
\end{tabular}




\begin{tabular}{|c|c|c|c|c|c|c|c|c|c|c|c|}
\hline Inst. & $|V|-|A|-|E|$ & $\left|A_{R}\right|$ & $\left|V_{R}\right|$ & $\left|V_{A C V R P}\right|$ & T.D. & $d_{1}$ & $K_{1}$ & Time $_{1}$ & $d_{2}$ & $K_{2}$ & $\mathrm{Time}_{2}$ \\
\hline I261 & $100-220-80$ & 88 & 2 & 253 & 10764 & 12723 & 11 & 643.9 & 11708 & 6 & 424.9 \\
\hline I 262 & $120-260-80$ & 104 & 3 & 273 & 12387 & 15312 & 13 & 529.4 & 14127 & 7 & 285.35 \\
\hline I 263 & $140-330-80$ & 132 & 2 & 299 & 14801 & 18469 & 15 & 1370.78 & 16110 & 8 & 552.23 \\
\hline $\mathrm{I} 264$ & $160-370-80$ & 148 & 11 & 359 & 16695 & 22098 & 17 & 1434.42 & 18966 & 9 & 651.6 \\
\hline I 265 & $180-400-80$ & 160 & 10 & 361 & 18031 & 22641 & 19 & 1089.58 & 19829 & 10 & 793.31 \\
\hline I266 & $200-420-80$ & 168 & 9 & 363 & 18409 & 23206 & 19 & 1685.57 & 20366 & 10 & 1026.85 \\
\hline $\mathrm{I} 271$ & $100-220-100$ & 88 & 2 & 293 & 12705 & 12708 & 13 & 912.82 & 11754 & 7 & 461.76 \\
\hline $\mathrm{I} 272$ & $120-260-100$ & 104 & 3 & 315 & 14161 & 15851 & 15 & 784.83 & 14099 & 8 & 490.66 \\
\hline $\mathrm{I} 273$ & $140-330-100$ & 132 & 5 & 353 & 16462 & 18388 & 17 & 1280.67 & 16269 & 9 & 1086.19 \\
\hline I 274 & $180-400-100$ & 160 & 9 & 406 & 19041 & 23053 & 20 & 1927.5 & 20180 & 10 & 1489.17 \\
\hline $\mathrm{I} 275$ & $200-420-100$ & 168 & 8 & 399 & 19914 & 29504 & 20 & 4160.04 & 23017 & 10 & 2203.86 \\
\hline I 281 & $200-600-60$ & 180 & 9 & 382 & 23593 & 25022 & 18 & 4449.39 & 20696 & 9 & 1819.96 \\
\hline I 282 & $200-600-80$ & 180 & 13 & 452 & 19844 & 26271 & 20 & 5636.98 & 223670 & 10 & 3160.30 \\
\hline I 283 & $200-600-100$ & 180 & 6 & 440 & 20324 & 24640 & 21 & 2681.01 & 21966 & 11 & 1802.93 \\
\hline I 284 & $200-600-120$ & 180 & 8 & 463 & 20555 & 26060 & 21 & 3841.40 & 23267 & 11 & 2136.15 \\
\hline I 285 & $200-600-140$ & 180 & 7 & 497 & 21577 & 27645 & 22 & 5756.68 & 24330 & 11 & 3655.87 \\
\hline I 286 & $200-600-160$ & 180 & 7 & 548 & 25148 & 25999 & 26 & 5966.49 & 23482 & 13 & 3879.16 \\
\hline I291 & $225-650-60$ & 195 & 19 & 439 & 21355 & 27266 & 22 & 2306.38 & 23901 & 11 & 2337.94 \\
\hline I292 & $225-650-80$ & 195 & 15 & 470 & 22727 & 29510 & 23 & 4450.06 & 248090 & 12 & 2900.06 \\
\hline I 293 & $225-650-100$ & 195 & 12 & 494 & 23987 & 28549 & 25 & 3187.86 & 25247 & 12 & 5064.86 \\
\hline I 294 & $225-650-120$ & 195 & 11 & 507 & 23593 & 31508 & 24 & 4747.81 & 27241 & 12 & 4546.69 \\
\hline I295 & $225-650-140$ & 195 & 6 & 502 & 23593 & 30096 & 24 & 4434.57 & 26147 & 12 & 5327.40 \\
\hline I 296 & $225-650-160$ & 195 & 5 & 538 & 24429 & 30333 & 25 & 5490.39 & 267850 & 13 & 5215.72 \\
\hline I301 & $250-700-60$ & 210 & 24 & 484 & 22346 & 28475 & 23 & 3718.08 & 247730 & 12 & 1945.99 \\
\hline I302 & $250-700-80$ & 210 & 19 & 484 & 22746 & 32558 & 23 & 4312.41 & 27064 & 12 & 2631.71 \\
\hline I303 & $250-700-100$ & 210 & 18 & 521 & 24925 & 29632 & 26 & 5427.05 & 25985 & 13 & 3103.63 \\
\hline I304 & $250-700-120$ & 210 & 7 & 490 & 23071 & 30786 & 24 & 3635.21 & 27128 & 12 & 2674.20 \\
\hline I305 & $250-700-140$ & 210 & 11 & 563 & 24875 & 374830 & 25 & 10734.52 & 297940 & 13 & 4286.82 \\
\hline I306 & $250-700-160$ & 210 & 11 & 604 & 27643 & 34824 & 28 & 9304.83 & 29962 & 14 & 6554.05 \\
\hline I311 & $270-750-60$ & 225 & 25 & 503 & 23948 & 30713 & 25 & 3003.68 & 29216 & 12 & 5038.99 \\
\hline I312 & $270-750-80$ & 225 & 28 & 599 & 27111 & 32940 & 28 & 4606.29 & 28394 & 14 & 5215.76 \\
\hline I313 & $270-750-100$ & 225 & 16 & 543 & 26907 & 33859 & 28 & 5026.30 & 29348 & 14 & 3865.43 \\
\hline I314 & $270-750-120$ & 225 & 13 & 548 & 25044 & 30692 & 26 & 4782.07 & 27320 & 13 & 4545.20 \\
\hline I315 & $270-750-140$ & 225 & 14 & 602 & 27722 & 36708 & 28 & 9063.87 & 306540 & 14 & 7840.02 \\
\hline I316 & $270-750-160$ & 225 & 9 & 623 & 27771 & 39925 & 28 & 11808.50 & 32859 & 14 & 9424.87 \\
\hline
\end{tabular}

\section{References}

Bautista, J., \& Pereira, J. (2004). Ant Algorithms for Urban Waste Collection Routing. Lecture Notes in Computer Science, 3172, 3020-3033.

Bautista, J., Fernández, E., \& Pereira, J. (2008). Solving an Urban Waste Collection Problem Using Ants Heuristics. Computers $\&$ Operations Research, 35, 302-309. 
Belenguer, J.M., Benavent, E., Lacomme, P., \& Prins, C. (2006). Lower and Upper Bounds for the Mixed Capacitated Arc Routing Problem. Computers 85 Operations Research, 33, 3363-3383.

Benavent, E., \& Soler, D. (1999). The Directed Rural Postman Problem with Turn Penalties. Transportation Science 33, 408-418.

Bodin, L., Fagin, G., Welebny, R., \& Greenberg, J. (1989). The Design of a Computerized Sanitation Vehicle Routing and Scheduling for the Town of Oyster Bay, New York. Computers \&S Operations Research, 16, 45-54.

Clossey, J., Laporte, G., \& Soriano, P. (2001). Solving Arc Routing Problems with Turn Penalties. Journal of the Operational Research Society, 52, 433-439.

Corberán, A., Martí, R., Martínez, E., \& Soler, D. (2002). The Rural Postman Problem on Mixed Graphs with Turn Penalties. Computers \& Operations Research, 29, 887-903.

De Franceschi, R., Fischetti, M., \& Toth, P. (2006). A New ILP-Based Refinement Heuristic for Vehicle Routing Problems. Mathematical Programming Series B, 105, 471-499.

Fischetti, M., Toth, P., \& Vigo, D. (1994). A Branch-and-Bound Algorithm for the Capacitated Vehicle Routing Problem on Directed Graphs. Operations Research, 42, 846-859.

Ghiani, G., \& Improta, G. (2000). An Efficient Transformation of the Generalized Vehicle Routing Problem. European Journal of Operational Research, 122, 11-17.

Jansen, K. (1993). Bounds for the General Capacitated Routing Problem. Networks, 23, 165-173.

Nagata, Y., \& Bräysy, O. (2009). Edge Assembly Based Memetic Algorithm for the Capacitated Vehicle Routing Problem. Networks, 54, 205-215.

Pandit, R., \& Muralidharan, B. (1995). A Capacitated General Routing Problem on Mixed Networks. Computers $\& 5$ Operations Research, 22, 465-478.

Perrier, N., Langevin, A., \& Amaya, C.V. (2008). Vehicle Routing for Urban Snow Plowing Operations. Transportation Science, 42, 44-56.

Pessoa, A., Poggi, M., \& Uchoa,E. (2007). Robust Branch-Cut-and-Price Algorithms for Vehicle Routing Problems. Presented at Route 2007 (International Work- 
shop on Vehicle Routing and Transportation), Jekyll Island, Georgia.

Roy, S., \& Rousseau, J.M. (1989). The Capacitated Canadian Postman Problem. INFOR, 27, 58-73.

Soler, D., Martínez, E., \& Micó, J.C. (2008). A Transformation for the Mixed General Routing Problem with Turn Penalties. Journal of the Operational Research Society, 59, 540-547.

Soler, D., Albiach, J., \& Martínez, E. (2009). A Way to Optimally Solve a TimeDependent Vehicle Routing Problem with Time Windows. Operations Research Letters, $37,37-42$.

Vigo, D. (1996). A Heuristic Algorithm for the Asymmetric Capacitated Vehicle Routing Problem. European Journal of Operational Research, 89, 108-126. 